\title{
Endothelial C3a receptor mediates vascular inflammation and blood-brain barrier permeability during aging
}

\author{
Nicholas E. Propson, ${ }^{1,2}$ Ethan R. Roy, ${ }^{2}$ Alexandra Litvinchuk, ${ }^{2}$ Jörg Köhl, ${ }^{3}$ and Hui Zheng ${ }^{1,2,4}$ \\ 'Department of Molecular and Cellular Biology, and ${ }^{2}$ Huffington Center on Aging, Baylor College of Medicine, Houston, Texas, USA. Innstitute for Systemic Inflammation Research, Center for Infectiology \\ and Inflammation Research Lübeck, University of Lübeck, Lübeck, Germany. ${ }^{4}$ Department of Molecular and Human Genetics, Baylor College of Medicine, Houston, Texas, USA.
}

\begin{abstract}
Dysfunction of immune and vascular systems has been implicated in aging and Alzheimer disease; however, their interrelatedness remains poorly understood. The complement pathway is a well-established regulator of innate immunity in the brain. Here, we report robust age-dependent increases in vascular inflammation, peripheral lymphocyte infiltration, and blood-brain barrier (BBB) permeability. These phenotypes were subdued by global inactivation and by endothelial cell-specific ablation of C3ar1. Using an in vitro model of the BBB, we identified intracellular $\mathrm{Ca}^{2+}$ as a downstream effector of $\mathrm{C} 3 \mathrm{a} / \mathrm{C} 3 \mathrm{aR}$ signaling and a functional mediator of vascular endothelial cadherin junction and barrier integrity. Endothelial C3ar1 inactivation also dampened microglia reactivity and improved hippocampal and cortical volumes in the aging brain, demonstrating a crosstalk between brain vasculature dysfunction and immune cell activation and neurodegeneration. Further, prominent C3aR-dependent vascular inflammation was also observed in a tau-transgenic mouse model. Our studies suggest that heightened $\mathrm{C} 3 \mathrm{a} / \mathrm{C} 3 \mathrm{aR}$ signaling through endothelial cells promotes vascular inflammation and BBB dysfunction and contributes to overall neuroinflammation in aging and neurodegenerative disease.
\end{abstract}

\section{Introduction}

The natural aging process includes functional and structural changes within the brain $(1,2)$, and these changes have been shown to play a role in decreased neural stem cell fitness, altered cognition, and increased susceptibility for neurodegenerative disease $(1,3,4)$. One such age-dependent change with potentially causal association with both normal decline and disease is dysfunction of the blood-brain barrier (BBB). The BBB is composed of endothelial cells, astrocytes, and pericytes, and an intact BBB is essential for brain health (5). Loss of vessel integrity is thought to drive BBB dysfunction and can be found in numerous neurological disease conditions, namely traumatic brain injury (6) and stroke (7), and often comorbidly in neurodegeneration (8). However, the mechanisms controlling changes in brain vasculature and their consequence to CNS function, in particular during aging, remain ill-defined.

Recent work has demonstrated a vascular component to age-related changes in the brain, deterioration in cognition, and eventual dementia $(9,10)$. Furthermore, evidence has been reported that vascular inflammation, marked by increased endothelial expression of vascular cell adhesion molecule VCAM1, stokes CNS aging by decreasing neural stem cell numbers and increasing microglial reactivity (11). Elevated levels of VCAM1 have also been found to correlate with Parkinson disease severity (12), and, recently, lymphocytes known to bind VCAM1 in

Conflict of interest: The authors have declared that no conflict of interest exists. Copyright: (๖) 2021, American Society for Clinical Investigation.

Submitted: June 3, 2020; Accepted: September 23, 2020; Published: January 4, 2021.

Reference information: J Clin Invest. 2021;131(1):e140966.

https://doi.org/10.1172/JCl140966 brain vasculature were found in aged patients and the brains of patients with Alzheimer disease $(13,14)$. Single-cell transcriptomic analysis of hippocampal brain endothelial cells has shown that age-related changes in these cells are rooted in responses to innate inflammatory cues, hypoxic stimuli, and oxidative stress (15). Together, these studies suggest a significant inflammatory transition in brain vasculature with age and the potential for a causal connection to CNS diseases.

Some studies of blood plasma components have implicated circulating factors in maintaining or diminishing brain health during aging (16), and others exploring local inflammatory cues in the CNS have highlighted the inherent capacity of glia to modulate neuroinflammation (17). One of the primary innate immune-signaling mechanisms involved in neuroinflammation is the complement pathway. Complement components are expressed by cells of the CNS and are reported to influence CNS aging and neurodegenerative disease progression (18). In particular, the complement component C3 is capable of potentiating age-related and neurodegenerative changes in the CNS (19-22). The active signaling peptide of $\mathrm{C} 3, \mathrm{C} 3 \mathrm{a}$, is released via cleavage by the extracellular enzyme C3 convertase. Once cleaved, C3a signals through its cognate receptor $\mathrm{C} 3 \mathrm{aR}$, which has been detected on microglia (23), choroid plexus epithelium (24), and vascular endothelial cells (25) in the brain. C3 is upregulated in astrocytes during aging and disease $(22,26)$, and the intimate relationship of astrocytes with the $\mathrm{BBB}$ supports the premise that $\mathrm{C} 3$ produced by these cells may play a direct role in age-related changes in brain vasculature.

Using in vivo and in vitro models, we identified a mechanism by which the C3a/C3aR signaling axis modulated VCAM1 expression, influenced peripheral immune cell infiltration, 
A

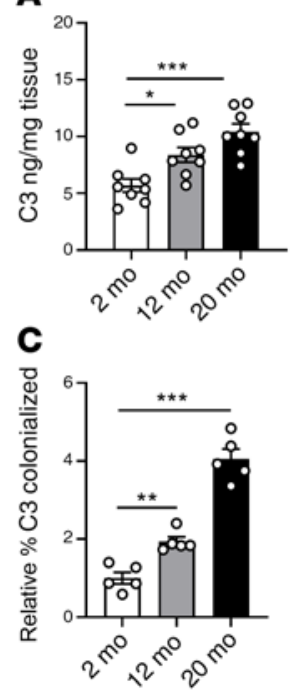

$\mathbf{F}$

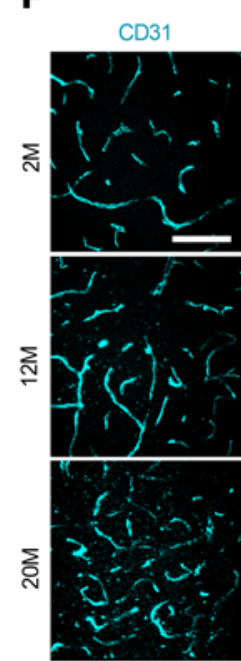

B

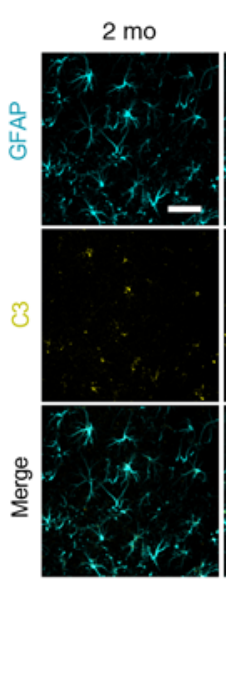

D

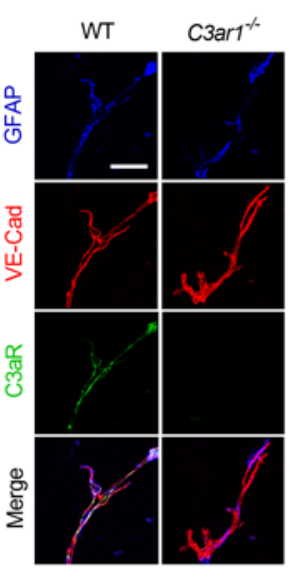

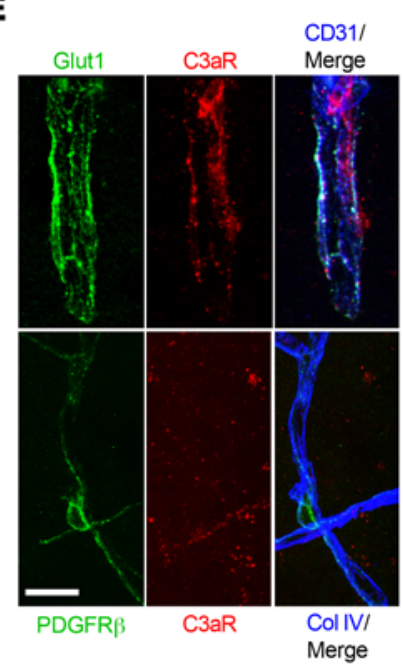

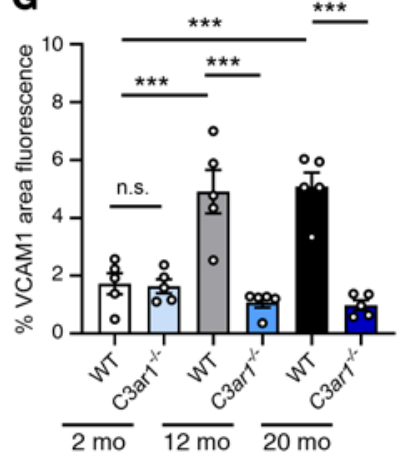

G
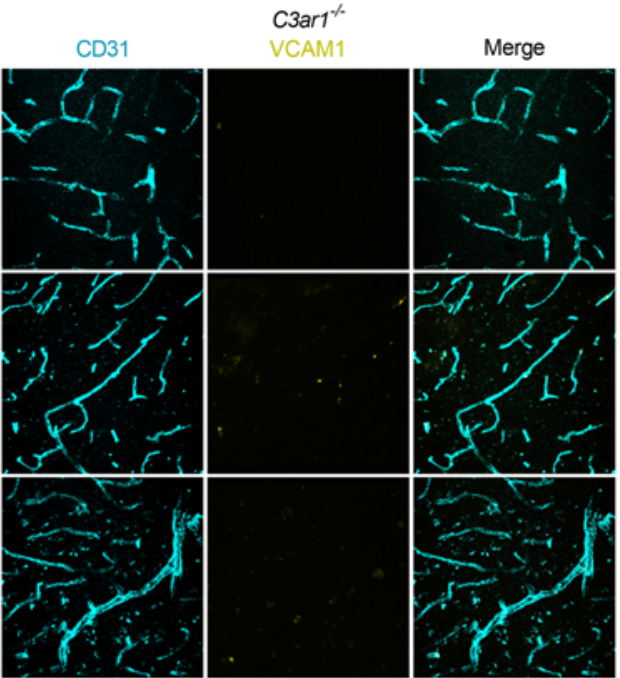
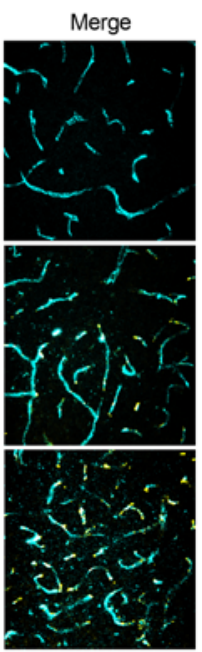
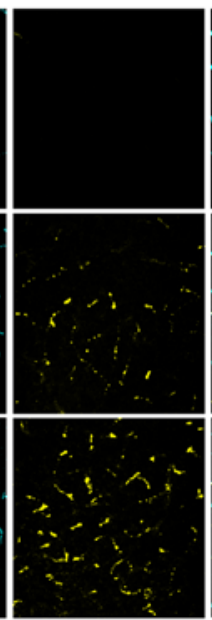
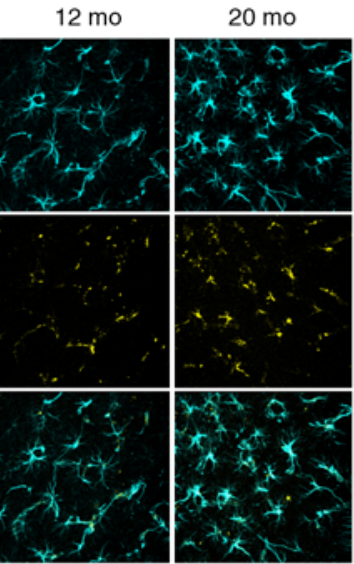

Figure 1. C3a/C3aR signaling regulates age-associated endothelial VCAM1 expression. (A) ELISA measurement of C3 levels in WT mouse brain lysates at 2 , 12, and 20 months ( $n=8$ /group). (B) Immunofluorescence staining using anti-GFAP and anti-C3 antibodies demonstrated localization of C3 to astrocytes. (C) Quantification confirming increased C3 staining within GFAP ${ }^{+}$astrocytes in the hippocampus with age $(n=5 /$ age). (D) Triple immunostaining of isolated vessels from WT and $\mathrm{CZar1}^{--}$brains using anti-GFAP, anti-VE-cadherin, and anti-C3aR antibodies showing positive C3aR staining along endothelial cell surface that was not present in C3ar1 ${ }^{-/-}$vessels. (E) Triple immunostaining of brain tissue with anti-Clut1, anti-C3aR, and anti-CD31 or anti-PDCFR- $\beta$, anti-C3aR, and anti-Col IV, demonstrating expression of C3aR on brain endothelial cells but not pericytes. (F and $\mathbf{G}$ ) Immunofluorescence staining and quantification using anti-CD31 and anti-VCAM1 antibodies of WT or C3ar1-- mouse cortices at 2, 12, and 20 months demonstrated an increase in VCAM1 with age in WT mice, but VCAM1 was rescued in the absence of C3aR. All data represent the mean \pm SEM. Significance was calculated using 1-way ANOVA with Tukey's post hoc test $\left({ }^{*} P<0.05\right.$, ${ }^{* *} P<$ $\left.0.01,{ }^{* * *} P<0.001\right)$. Scale bars: $20 \mu \mathrm{m}$ (B), $10 \mu \mathrm{m}$ (D), $15 \mu \mathrm{m}$ (E), and $50 \mu \mathrm{m}$ (F).

altered vascular morphology, increased BBB permeability, and potentiated microglial reactivity and neurodegeneration in aged mice. We further showed that this C3aR-dependent endothelial phenotype was exacerbated in PS19 tau-transgenic mice, a model in which elevated C3/C3aR signaling was shown to modulate CNS inflammation and tau pathology (22). This study identified complement signaling as a key mediator of vascular dysfunction in brain aging and disease.

\section{Results}

C3a/C3aR signaling regulates age-associated endothelial VCAM1 expression and immune cell infiltration. C3 mRNA has been shown to be upregulated in aged astrocytes $(20,26)$. To corroborate this finding, we measured C3 protein in lysates of mouse brains at 2, 12, and 20 months of age by ELISA. We detected a significant increase at 12 months, with a further increase at 20 months, over levels detected in the young mice (Figure 1A). Consistent with our earlier reports in disease models $(21,22)$, coimmunofluorescent labeling of the hippocampus showed C3 expression predominantly colocalized in $\mathrm{GFAP}^{+}$astrocytes, where its levels were elevated during aging (Figure 1, B and C, and Supplemental Figure 1A; supplemental material available online with this article; https://doi. org/10.1172/JCI140966DS1). Increased C3 mRNA was further validated in FACS-sorted aged astrocytes using our previously 
published method (27) (Supplemental Figure 1B). To examine the expression of $\mathrm{C} 3 \mathrm{aR}$ in brain vasculature, we isolated vessels from WT and $\mathrm{C} \mathrm{arl}^{-/-}$mouse brains (28) and immunostained them with antibodies against GFAP, VE-cadherin, and C3aR. Positive C3aR staining could be readily detected in VE-cadherin ${ }^{+}$brain endothelial cells in WT but not $\mathrm{C} \mathrm{arr}^{-/-}$vessels (Figure 1D). High-resolution confocal imaging analysis of CD31, Glut1, and C3aR on mouse brain vasculature revealed a greater polarization of $\mathrm{C} 3 \mathrm{aR}$ toward the basolateral surface, whereas Glut1 localized predominantly toward the vessel lumen (Figure 1E and Supplemental Figure 1C). In contrast to the endothelial expression, costaining for C3aR and the pericyte marker PDGFR- $\beta$ did not detect appreciable colocalization (Figure 1E). Flow cytometry analysis of human brain microvascular endothelial cells (HBMECs) showed high levels of positivity for VE-cadherin (80.9\%) and Glut1 (94.2\%), as expected, and this positivity was also observed for $\mathrm{C} 3 \mathrm{aR}$, although to a lesser degree (34.3\%) (Supplemental Figure 1D). Together, these results established C3aR expression in brain vascular endothelial cells and supported a signaling axis involving astroglial C3 and endothelial C3aR at the BBB.

To assess the functional role of this signaling pathway, we first analyzed the expression of VCAM1 because it has been implicated in complement-mediated activation of brain endothelial cells in LPS-induced neuroinflammation (29) and ischemia models (30). Coimmunofluorescent labeling of brain cortical vasculature revealed that VCAM1 expression was restricted to $\mathrm{CD} 31^{+}$vasculature, where its levels were increased with age in WT mice but not in C3ar1-null mice (Figure 1, F and G). A similar reduction in vascular VCAM1 was also seen after treating 2-, 12-, and 20-month-old mice with the C3aR antagonist (C3aRA) SB290157 (Supplemental Figure 2, A and $\mathrm{B})$, further confirming our genetic study and validating the inhibitory effect of C3aRA. The same was true when hippocampal samples were analyzed (Supplemental Figure 2C). We then performed deeper analysis by measuring Vcam1 mRNA levels in FACS-sorted mouse brain endothelial cells from 2-, 12-, and 20-month-old cohorts treated with vehicle or C3aRA, which showed increased Vcam1 expression with age in vehicle-treated samples but blunted expression with C3aRA treatment (Supplemental Figure 2D).

To substantiate this signaling pathway in human cells and to test a direct effect of $\mathrm{C} 3 \mathrm{a} / \mathrm{C} 3 \mathrm{aR}$ signaling, we treated primary HBMECs with recombinant human C3a, with or without C3aRA, and found a robust increase in Vcam1 expression by $\mathrm{C} 3 \mathrm{a}$ treatment, which was quelled in the presence of C3aRA (Supplemental Figure 2E). Interestingly, other adhesion molecules, namely Sele and Icam1, were not significantly changed by this treatment (Supplemental Figure 2, F and G). Additionally, similar ICAM1 immuno-intensities (Supplemental Figure 2, H-J) and Icam1 mRNA levels (Supplemental Figure 2K) were detected in 2- and 20-month-old mouse brains. Together, these data support both the necessity and sufficiency of $\mathrm{C} 3 \mathrm{a}$ signaling in modulating VCAM1 expression in brain endothelial cells.

Next, we examined the functional consequence of C3-mediated VCAM1 upregulation. It has been previously shown that increased numbers of peripheral lymphocytes are found in the aged brain (31) and reside in the neural stem cell niche of the subventricular zone (13). Because adhesion molecules regulate the process of rolling adhesion and extravasation of immune cells, we hypothesized that increased brain VCAM1 expression in vasculature during aging may be associated with increased peripheral immune cell infiltration. Using FACS to discriminate CD $45^{\mathrm{hi}}$ / CD $11 b^{-}$lymphocytes from $\mathrm{CD} 45^{\mathrm{hi} / \mathrm{mid}} / \mathrm{CD} 11 \mathrm{~b}^{+}$monocytes and microglia in dissociated brain tissues, we found increasing proportions of CD $45^{\text {hi }} / \mathrm{CD}_{11} \mathrm{~b}^{-}$infiltrates at 12 and 20 months compared with 2-month-old controls (Figure 2, A and B). In contrast, monocytes were not significantly changed with age (Supplemental Figure 3A). We next asked whether, in addition to reducing VCAM1 expression levels, blocking C3aR signaling could reduce age-related lymphocyte infiltration. Flow cytometry analysis of 12- to 14-month-old $\mathrm{C}_{3 \mathrm{arr}^{-/-}}$mice revealed a reduction in the total percentage of $\mathrm{CD} 45^{\mathrm{hi}} / \mathrm{CD} 11 \mathrm{~b}$-infiltrating lymphocytes in the brain compared with WT controls (Figure 2, C and D), whereas monocytes were unaffected (Supplemental Figure 3B). Similar reductions in $\mathrm{CD} 45^{\mathrm{hi}} / \mathrm{CD} 11 \mathrm{~b}$-infiltrating lymphocytes but not monocytes were observed after treating 20-month-old WT mice with C3aRA (Supplemental Figure 3, C-E).

Given that acute neuroinflammation induces peripheral immune cell infiltration (29), we tested the role of C3aR in recruiting these cells after i.c.v. administration of LPS in 3- to 4-monthold WT and $\mathrm{C} \mathrm{arl}^{-/-}$mice (Supplemental Figure 4A). As expected, LPS challenge led to increased lymphocyte (Supplemental Figure 4B) and monocyte (Supplemental Figure 4C) infiltration in the brains of WT mice. In contrast, genetic ablation of C3ar1 blunted the infiltration of $\mathrm{CD} 45^{\text {hi }} / \mathrm{CD} 11 \mathrm{~b}^{-}$peripheral lymphocytes (Supplemental Figure 4B), whereas monocyte cell numbers were only marginally affected (Supplemental Figure 4C). Consistent with the specific regulation of VCAM1 by C3a/C3aR signaling, deletion of C3ar1 significantly suppressed Vcam 1 gene expression induced by LPS without affecting Sele and Icam 1 in FACS-sorted brain endothelial cells (Supplemental Figure 4D).

To assess whether LPS-induced acute neuroinflammation affects BBB permeability, we analyzed each group using a tail-vein injection of a BBB impenetrable TRITC-dextran dye (65-85 kDa). Interestingly, there was no significant increase in $\mathrm{BBB}$ permeability under acute neuroinflammatory stimuli that contributed to this infiltration event (Supplemental Figure 4E). To confirm the induction of neuroinflammation by LPS, we analyzed sorted microglia from the vehicle- and LPS-treated animals and found consistent microglial gene response signatures, as we have previously reported (27) (Supplemental Figure 4F).

Together, these results suggest that C3a acts through endothelial C3aR to promote VCAM1 expression, and that during both aging and acute neuroinflammation, this pathway plays a role in selective infiltration of lymphocytes into the brain.

$C D 8^{+} T$ cells preferentially infiltrate the aged brain. We next examined the lymphocyte subtypes infiltrating the aged brain and compared these with the cells of the aged spleen to better understand the global changes to lymphocyte composition in aged tissues. Dissociated cells from the brain or spleen of 20-month-old WT mice were immunostained using anti-CD45, anti-CD11b, anti-CD3e, anti-CD19, anti-CD8a, and anti-CD4 antibodies. After antibody staining, cells were analyzed by flow cytometry to differentiate monocytes, microglia, and lymphocytes (Figure $3 \mathrm{~A}$, left panels). Further subtyping was carried out to analyze $\mathrm{T}$ lymphocyte versus B lymphocyte composition (Figure 3A, middle 
A

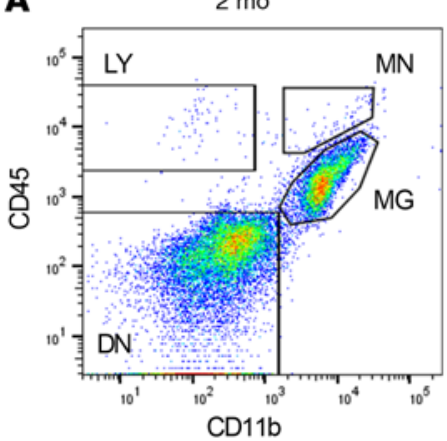

C

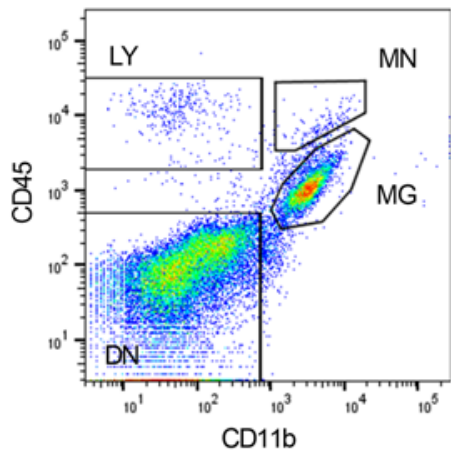

$12 \mathrm{mo}$

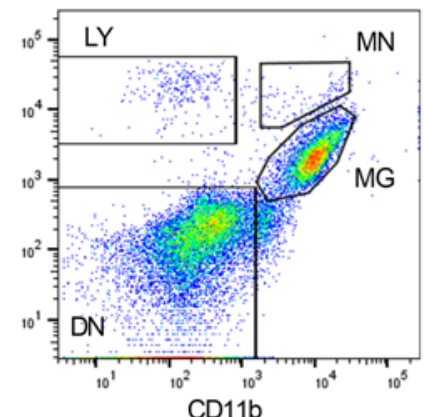

$\mathrm{CD} 11 \mathrm{~b}$

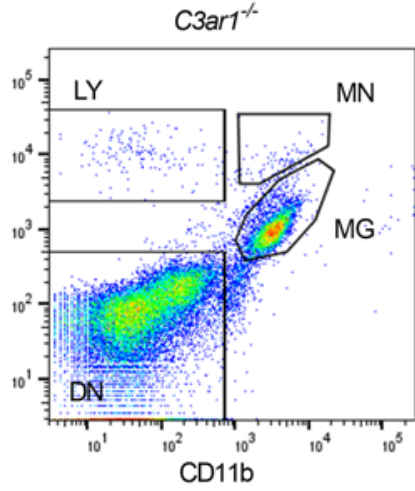

$20 \mathrm{mo}$

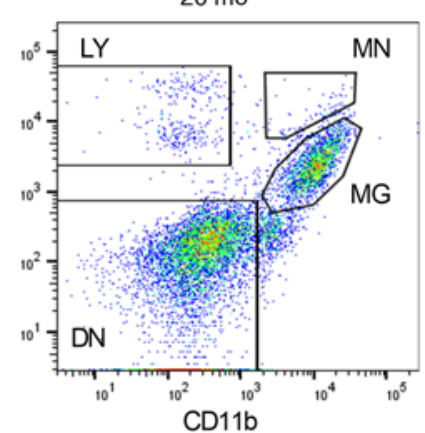

B

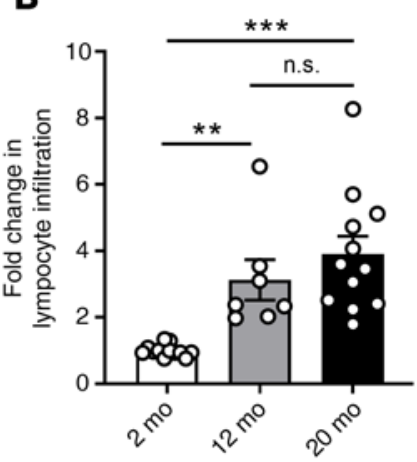

D

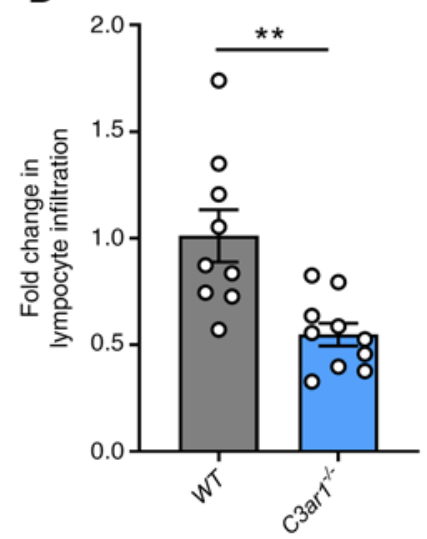

Figure 2. C3aR/VCAM1 axis promotes peripheral lymphocyte infiltration during aging. (A) Representative CD45 and CD11b flow cytometric plots and gating strategy of dissociated mouse brain at 2 months, 12 months, and 20 months. LY, lymphocyte; MN, monocyte; MG, microglia; DN, double-negative. (B) Flow cytometric analysis and quantification of percentage of infiltrating lymphocytes ( 2 months $n=12,12$ months $n=7$, 20 months $n=12$ ) showed age-related increase. (C

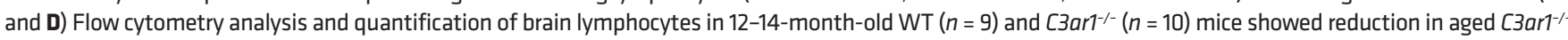
mice. All data represent the mean \pm SEM. Significance was calculated using 1-way ANOVA with Tukey's post hoc test $\left(^{* *} P<0.01\right.$, $\left.{ }^{* * *} P<0.001\right)$.

panels), and T lymphocyte subtyping to differentiate $\mathrm{CD}^{+}$versus $\mathrm{CD}^{+} \mathrm{T}$ cells (Figure 3A, right panels). In 20-month-old WT mice, we found that infiltrating lymphocytes were predominantly $\mathrm{CD}^{+}(75 \%)$ versus CD19+ $(10 \%)$, and analysis of matched spleens showed significantly more CD19+ $(65 \%)$ cells versus CD3 ${ }^{+}(25 \%)$ cells (Figure 3B), suggesting that the aged brain preferentially recruited $\mathrm{CD}^{+} \mathrm{T}$ cells compared with the aged spleen. Further analysis of brain $\mathrm{CD}^{+} \mathrm{T}$ cells showed that the majority were $\mathrm{CD} 8^{+}$ $\mathrm{T}$ cells $(75 \%)$ instead of $\mathrm{CD}^{+} \mathrm{T}$ cells $(15 \%)$, which also inversely correlated with the peripheral splenic tissue, where $\mathrm{CD} 4^{+} \mathrm{T}$ cells were significantly more abundant than $\mathrm{CD} 8^{+} \mathrm{T}$ cells (Figure $3 \mathrm{C}$ ). This data suggests that $\mathrm{CD} 8^{+} \mathrm{T}$ cells were preferentially recruited to the aged brain compared with other lymphocyte subsets.

To further evaluate whether this age-dependent change was specific to brain parenchyma, representing potential infiltration, we isolated the choroid plexus tissue from brain ventricles of 2- and 20-month-old mice prior to dissociation and performed similar analyses as stated above, using the spleen as a control. Using the same flow cytometry gating strategy, we saw increased numbers of $\mathrm{CD}^{+}$ and $\mathrm{CD}^{+}$lymphocytes only in aged brains, but not in aged choroid plexus tissue (Supplemental Figure 5, A-C) or the spleen (Supplemental Figure 5, D-F). These data suggest that the age-related CD8 ${ }^{+}$lymphocyte infiltration was specific to brain parenchyma and not present in other immune cell-rich regions of the brain or peripheral tissues.
To confirm that peripheral cells are recruited to and infiltrate the aged brain, we employed an $\mathrm{mT} / \mathrm{mG}$ reporter mouse model, which produces tdTomato under the ROSA26 locus (32). When crossed with Mx1-Cre mice and activated by peripheral poly I:C (polyinosinic:polycytidylic acid) treatment, Cre-responsive cells in the periphery recombined to express EGFP, generating a chimeric mouse (MXG) (Supplemental Figure 6A). Analysis of PBMCs confirmed that approximately $40 \%$ of PBMCs were converted to express EGFP in MXG mice (Supplemental Figure 6, B-D). To assess brain infiltration of $\mathrm{EGFP}^{+}$peripheral cells during aging, we injected mice with poly I:C at 2 months of age and allowed mice to age to 15 months. Flow cytometry analysis of dissociated brains showed that approximately half of infiltrating CD $45^{\text {hi }} / \mathrm{CD} 11 \mathrm{~b}^{-}$lymphocytes expressed EGFP in 15-month-old MXG brains (Supplemental Figure 6, E-G), demonstrating that lymphocytes in aged brains were peripherally derived. Confocal imaging of the aged brain tissue confirmed the presence of $\mathrm{EGFP}^{+}$-infiltrated peripheral immune cells along the basolateral surface of tdTomato $\mathrm{EGFP}^{-}$brain vessels, further indicating the peripheral origin of the cells (Supplemental Figure 6H).

To better understand the regional distribution of these $\mathrm{CD}^{+}$ infiltrates, we analyzed 2- and 20-month-old mouse brain tissue by immunostaining with antibodies against CD8 to mark the T cells of interest and collagen IV (Col IV) to mark the endothelial basement membrane (Figure 3, D and E). Besides the subventricular zone as 
A
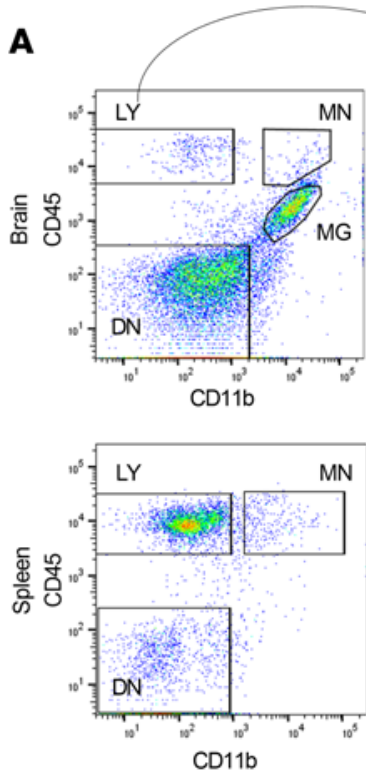

D
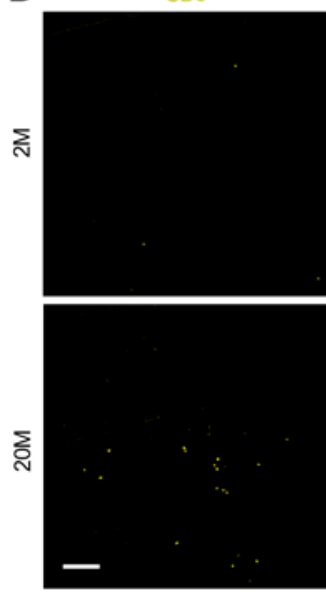

E

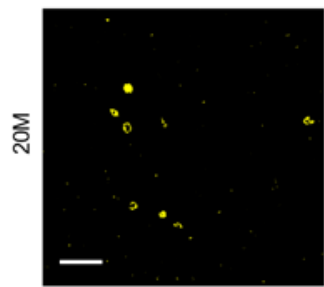

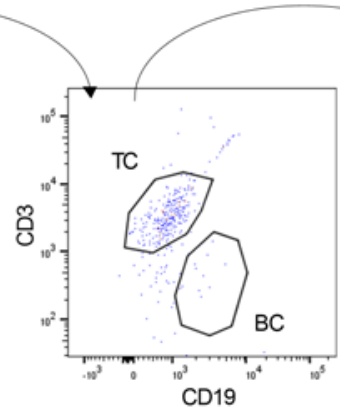

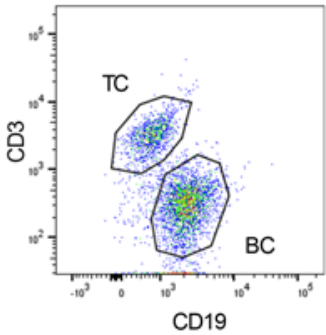

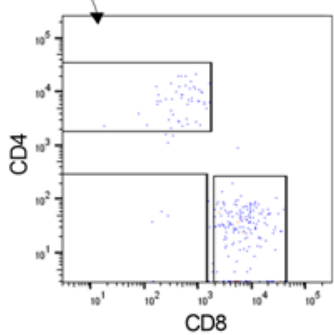

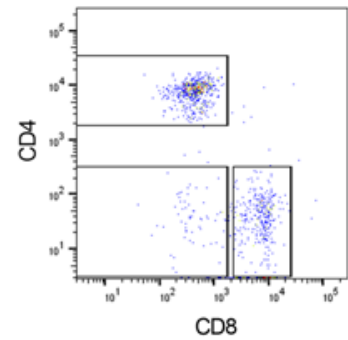

$\mathbf{F}$
B
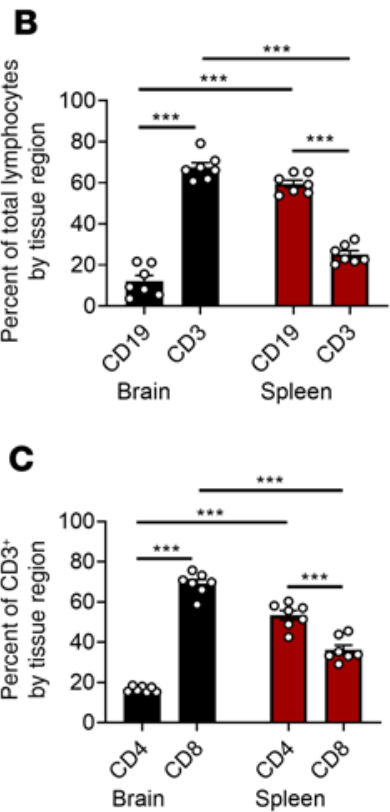

Col IV
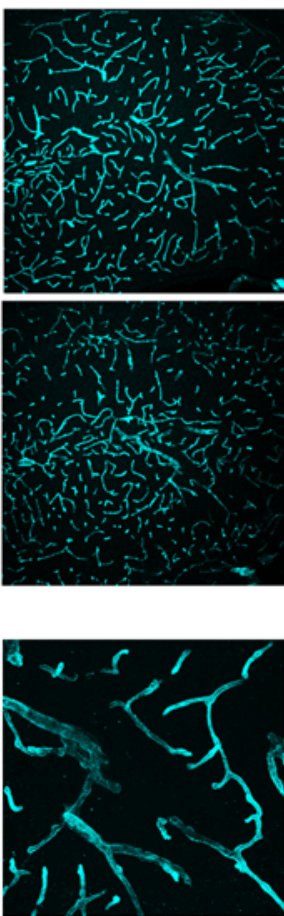

Merge

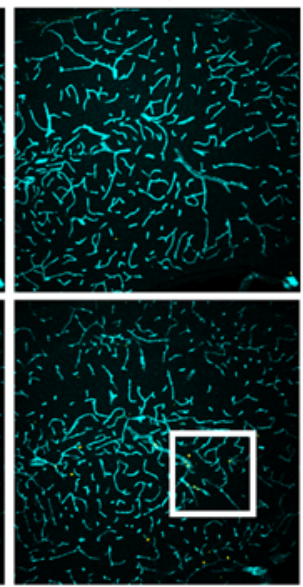

G

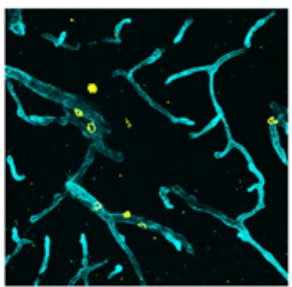

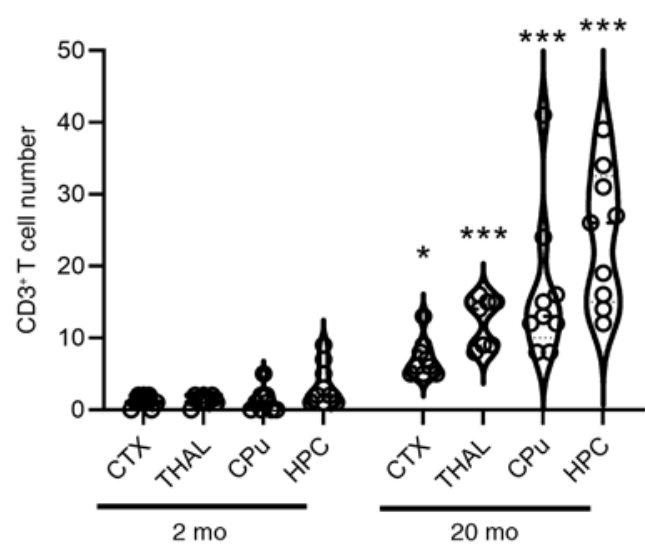
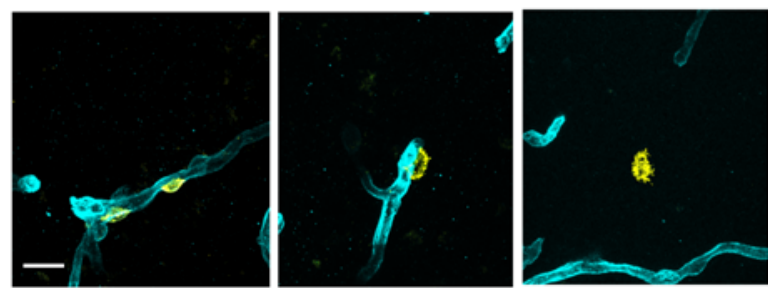

Figure 3. CD8+ $\mathbf{T}$ cells are preferentially recruited and infiltrate the aged brain. (A) Schematic for flow cytometry analysis of dissociated infiltrating cells in the 20-month-old brain or spleen using antibody staining against CD45, CD11b, CD3E, CD19, CD8a, and CD4. TC, T cells; BC, B cells. (B) Quantification of flow cytometry analysis showed predominantly CD3+ $\mathrm{T}$ cells in the brain compared with the spleen, which showed predominantly CD19+ B cells ( $n=7 /$ group). (C) Quantification of T cells showed the predominant subtype enriched in the brain was CD8+ $T$ cells compared with CD4 $4^{+} T$ cells in the spleen ( $n=7 /$ group). (D) Representative immunostaining of 2-month-old and 20-month-old brain tissue using anti-CD8a and anti-Col IV to determine regional immune cell infiltration within the brain. (E) Magnified image from $\mathbf{D}$ highlighting the infiltrated cell types that were counted for analysis. (F) Quantification of regional distribution of $C D 8^{+} T$ cell infiltrates in 4 major brain regions ( $n=4 /$ group, 2 tissue sections per mouse). CTX, cortex; THAL, thalamus; CPu, caudate putamen; HPC,hippocampus. (C) Representative images of 3 distinct stages of infiltration observed in all brain regions: perivascular residence (left panel), extravasation (middle panel), and parenchymal surveillance (right panel). Data in B and $\mathbf{C}$ represent the mean \pm SEM. Analysis was done using 1-way ANOVA with Tukey's post hoc test $\left.{ }^{* * *} P<0.001\right)$. Data in $\mathbf{F}$ are violin plots displaying medians and quartile ranges. Analysis was done using 2 -way ANOVA with Holm-Sidak post hoc test $\left({ }^{*} P<0.05,{ }^{* *} P<0.001\right)$. Scale bars: $100 \mu \mathrm{m}$ (D), $50 \mu \mathrm{m}$ (E), $10 \mu \mathrm{m}$ (C). 
previously reported (13), it was clear that CD8 T cells were present in other brain regions as well. Notably, the cortex, thalamus, caudate putamen, and the hippocampus all had a significant presence of CD8 T cells with age (Figure 3F). These cells were found either residing along the basolateral surface with colocalized Col IV (Figure 3G left panel), extravasating with minimally colocalized Col IV (Figure 3G middle panel), or fully extravasated into the tissue parenchyma (Figure $3 \mathrm{G}$ right panel). Overall, these findings demonstrated that during aging, peripherally derived $\mathrm{CD}^{+} \mathrm{T}$ cells preferentially infiltrated and took up residence inside the brain parenchyma in numerous tissue regions.

Inhibition of $C 3 a R$ rescues age-related changes in vascular morphology and $B B B$ permeability. We next determined the effect of $\mathrm{C} 3 \mathrm{a} / \mathrm{C} 3 \mathrm{aR}$ signaling on brain vascular morphology at different ages. We performed confocal imaging and $3 \mathrm{D}$ reconstruction of the vasculature, visualized by Col IV staining, and measured the average cross-sectional area of hippocampal capillaries by dividing the total $\mathrm{Col} \mathrm{IV}^{+}$volume by the total capillary length in each reconstructed image. Capillaries in young mice showed an average crosssectional area of approximately $60 \mu \mathrm{m}^{2}$, whereas capillaries in 12- and 20-month-old mice averaged approximately $40 \mu \mathrm{m}^{2}$ (Figure $4 \mathrm{~A}$ ). This reduction was partially but significantly rescued in 20-month-old $\mathrm{C} \mathrm{arl}^{-/-}$mice and in mice treated with C3aRA (Figure $4 \mathrm{~B})$. Analysis of each component of this measurement demonstrated a decrease in total vascular volume (Supplemental Figure 7A) and an increase in vessel length (Supplemental Figure 7B) during aging, both of which contributed to decreased average cross-sectional area. Additionally, $\mathrm{CD} 31^{+}$vessels showed a higher degree of tortuosity in the aged hippocampus, as previously defined by their corkscrew-like morphology $(33,34)$ (Figure $4 \mathrm{C}$, marked by rectangles). This phenomenon has been linked to decreased hemodynamic flow and hypoxia in affected brain regions $(33,34)$. The overall incidence of tortuous vessel segments was increased approximately 2- and 4-fold by 12 and 20 months, respectively, over that observed in 2-month-old mice (Figure 4, C and D). This vascular phenotype was rescued by both genetic ablation of $C 3$ arl and C3aRA treatment in 20-month-old mice (Figure 4, C and D). Thus, blocking C3aR significantly improved vascular morphology in aged mice.

Given the observed age-associated changes in lymphocyte infiltration and vascular morphology, we tested whether BBB integrity was affected by $\mathrm{C} 3 \mathrm{a} / \mathrm{C} 3 \mathrm{aR}$ signaling. We used a previously reported method allowing detection of peripherally administered fluorescent dextran leakage into the brain (35) (Figure 4E). At 2 months, there was almost no detection of fluorescent dextran in brain tissues, whereas there was a robust increase in fluorescent signal in 20-month-old brains, localized around lectin ${ }^{+}$vasculature (Figure 4E). Quantification of dextran levels in brain homogenates of 2-, 12-, and 20-month-old mice showed an age-dependent tracer leakage into the brain at 12 months ( 2-fold) and 20 months $(\sim 3-$ fold) (Figure 4F). Treating 20-month-old mice with C3aRA modestly but significantly reduced tracer leakage (Figure 4G).

To further characterize age-associated changes in BBB integrity, we examined VE-cadherin ${ }^{+}$intercellular junctions by confocal microscopy in vessels isolated from 2-, 12-, and 20-month-old mouse brains (28) with or without C3aRA treatment (Figure $4 \mathrm{H}$ and Supplemental Figure 7C). Quantification of immunoreactivity in large vessels (Figure 4I) and capillaries (Supplemental Figure
7D) revealed age-associated downregulation of VE-cadherin, with modest but significant rescue by C3aRA treatment. Consistent with this, gene expression analysis of FACS-sorted brain endothelial cells showed age-dependent reduction of Cdh5 (encoding VE-cadherin) at 12 and 20 months, along with reductions in Ocln, Tjp1, and Cldn5 mRNAs (Supplemental Figure 7E), reflecting impaired expression of junctional components. These age-dependent reductions were either trending or significantly restored with C3aRA treatment (Supplemental Figure 7E). Together, these data suggest that C3aR inhibition partially restored BBB integrity in aged brains.

C3a-mediated barrier disruption involves $\mathrm{Ca}^{2+}$ mobilization, cytoskeletal activation, and VE-cadherin disruption. To elucidate the mechanism of C3a-mediated BBB permeability in vitro, we employed the use of transendothelial electrical resistance (TEER) measurements. Using chopstick electrodes and an EVOM2 ohmmeter to measure the resistance of electrical current in an isolated system, we could directly test the effect of various molecules in an in vitro barrier model (Figure 5A). Our model contained a coculture of primary human astrocytes cultured for 2 days on the abluminal surface of a semipermeable membrane prior to the addition of HBMECs, which were cultured on the luminal surface for another 4 days, while TEER was monitored for optimal resistance prior to beginning treatment. We first validated the capability of this system to generate a reproducible barrier. Using the TEER as a readout, we compared resistance of a cell-free membrane with barriers formed by primary astrocytes or endothelial cells, or by endothelial cells cocultured with HeLa cells or primary astrocytes. We found that endothelial cells cocultured with astrocytes, but not with HeLa cells, significantly increased TEER (Supplemental Figure 8, A and B), demonstrating that astrocytes promoted BBB integrity in vitro.

To test the effect of C3aR signaling, we treated human endothelial cell-astrocyte cocultures with recombinant human C3a, and used IL-1 $\beta$ treatment, known to disrupt barrier integrity, as a positive control (36). After a 24-hour treatment, we found that C3a treatment resulted in a significant reduction of TEER, similar to IL-1 $\beta$ (Figure 5, B and C). C5a treatment had a marginal but not statistically significant effect (Supplemental Figure 8, C and D). C3a-induced barrier dysfunction was blocked when cultures were cotreated with C3aRA (Figure 5, B and C).

To further elucidate the mechanism of $\mathrm{C} 3 \mathrm{aR}$-mediated $\mathrm{BBB}$ permeability, we interrogated intracellular $\mathrm{Ca}^{2+}$ as a potential second messenger, given previous reports that activation of $\mathrm{C} 3 \mathrm{aR}$ triggers $\mathrm{Ca}^{2+}$ release (37). Treating the cocultures with ionomycin resulted in a drastic reduction of TEER (Supplemental Figure 8, E and F), suggesting $\mathrm{Ca}^{2+}$ release was sufficient to increase barrier permeability. Cotreatment with $\mathrm{C} 3 \mathrm{a}$ and the calcium chelator BAPTA-AM, to block $\mathrm{Ca}^{2+}$ signaling, led to a rescue of $\mathrm{C} 3 \mathrm{a}$-mediated TEER reduction to the level observed with C3aRA intervention (Figure 5, D and $\mathrm{E}$ ), suggesting that calcium release was the primary mechanism of barrier permeability downstream of $\mathrm{C} 3 \mathrm{a} / \mathrm{C} 3 \mathrm{aR}$ signaling.

Next, we aimed to dissect the connection between changes in VE-cadherin and $\mathrm{Ca}^{2+}$ that may result in increased barrier permeability downstream of C3aR activation. Previous work has shown that various endothelial cell lines respond to $\mathrm{C} 3 \mathrm{a}$ by forming actin stress fibers within the cell (25). After activation of the calciumdependent kinase calmodulin, myosin light chain (MLC) kinase is 

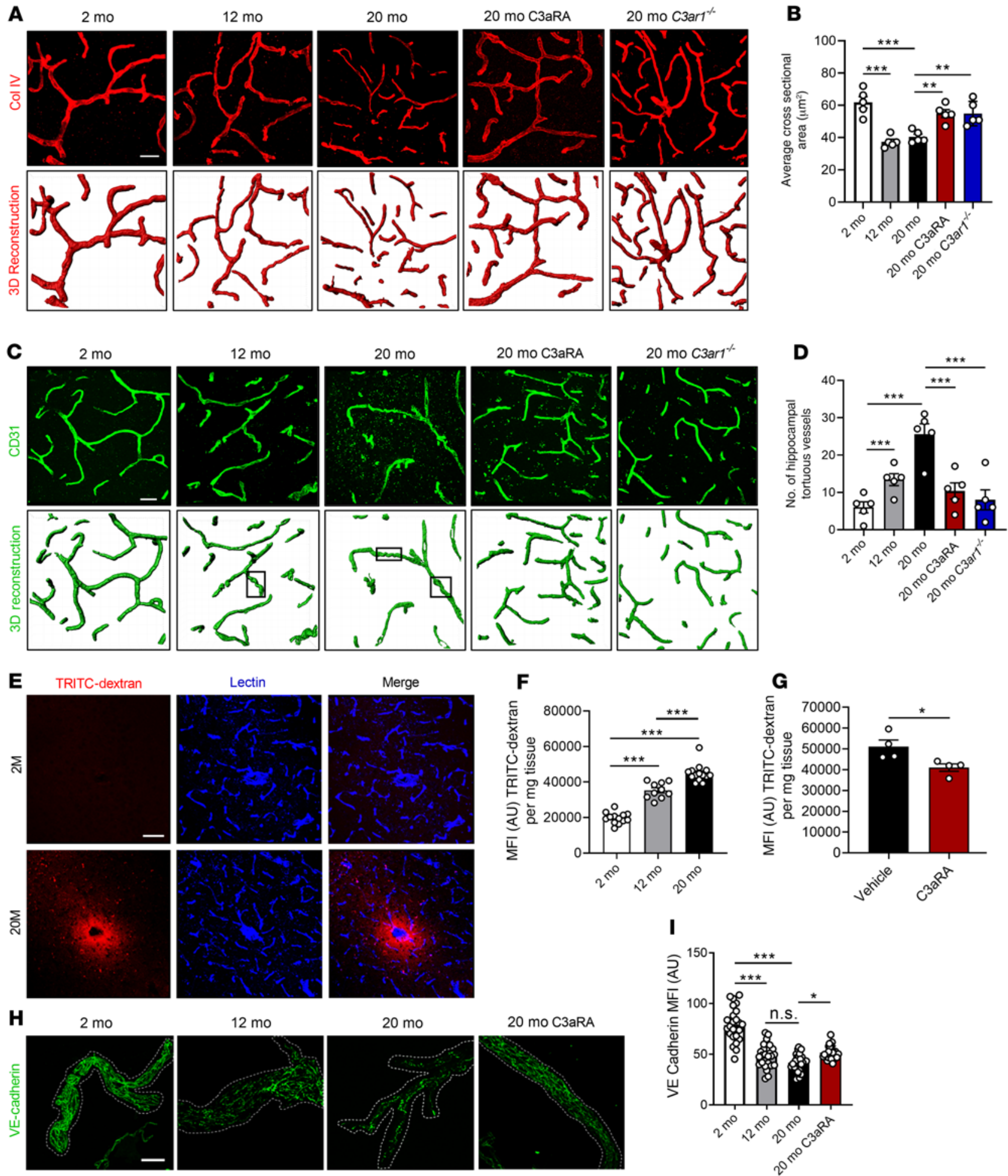

Figure 4. Inhibition of C3aR rescues age-related changes in vascular morphology and BBB permeability. (A) Representative collagen IV+ (Col IV) staining and IMARIS-aided 3D reconstruction of vasculature in hippocampal sections from 2-, 12-, and 20-month-old WT mice; 20-month-old WT mice treated with C3aRA; or 20-month-old C3ar1 ${ }^{-1-}$ mice. (B) Quantification of capillary average cross-sectional area in $\mathbf{A}$ ( $n=5 /$ group, 8 images per mouse). (C) Representative CD31 ${ }^{+}$staining and $3 \mathrm{D}$ reconstruction of hippocampal vasculature in 2-, 12-, and 20-month-old WT mice; 20-month-old WT mice treated with C3aRA; or 20-month-old C3ar1 ${ }^{-1-}$ mice. Representative tortuous vessels are marked by rectangles. (D) Quantification of number of tortuous vessels per hippocampal areas ( $n=5 /$ group, 8 images per mouse). (E) Representative lectin and TRITC-dextran colabeling in 2- and 20-month-old hippocampi. (F) Quantification of TRITC-dextran MFI from brain lysates of 2-month-old ( $n=13), 12$-month-old $(n=10)$, and 20-month-old $(n=14)$ mice. (G) Quantification of TRITC-dextran MFI of 20-month-old mice treated with vehicle or C3aRA ( $n=4 /$ group). (H) Representative image of vessels isolated from 2- and 12-month-old mice or 20-month-old mice treated with vehicle or C3aRA and stained with anti-VE-cadherin. (I) Quantification of VE-cadherin staining showed reduced VE-cadherin expression in 12- and 20-month-old mice, which was partially rescued in 20-month-old mice treated with C3aRA ( $n=5 /$ group, 5 vessel fragments/mouse). All data represent the mean \pm SEM. Significance was calculated using 1-way ANOVA with Tukey's post hoc test $\left({ }^{*} P<0.05,{ }^{* *} P<0.01,{ }^{* * *} P<0.001\right)$. NScale bars: $20 \mu \mathrm{m}(\mathbf{A}$ and $\mathbf{C}) ; 50 \mu \mathrm{m}$ (E). 
A

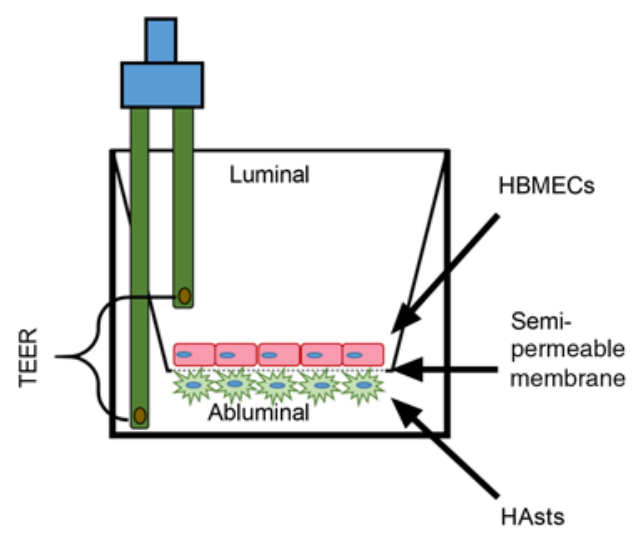

B

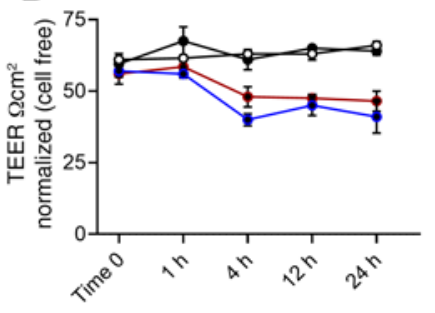

$\multimap$ Vehicle $\quad-\mathrm{C} 3 \mathrm{a}$

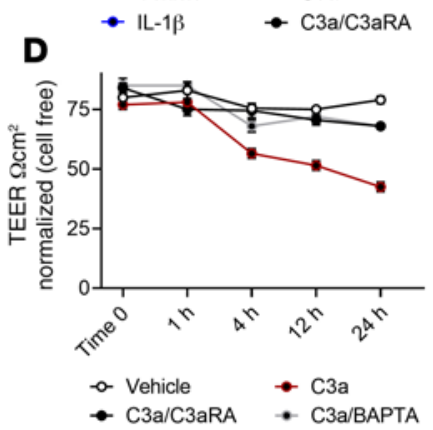

C

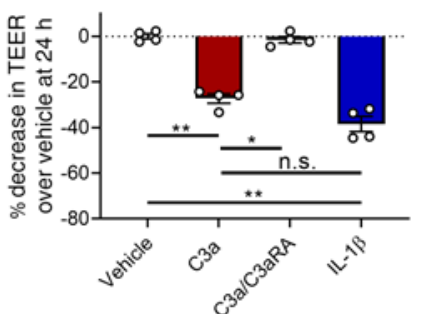

E

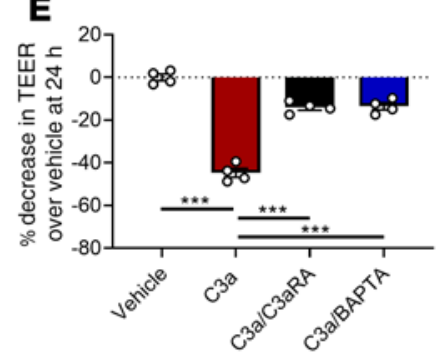

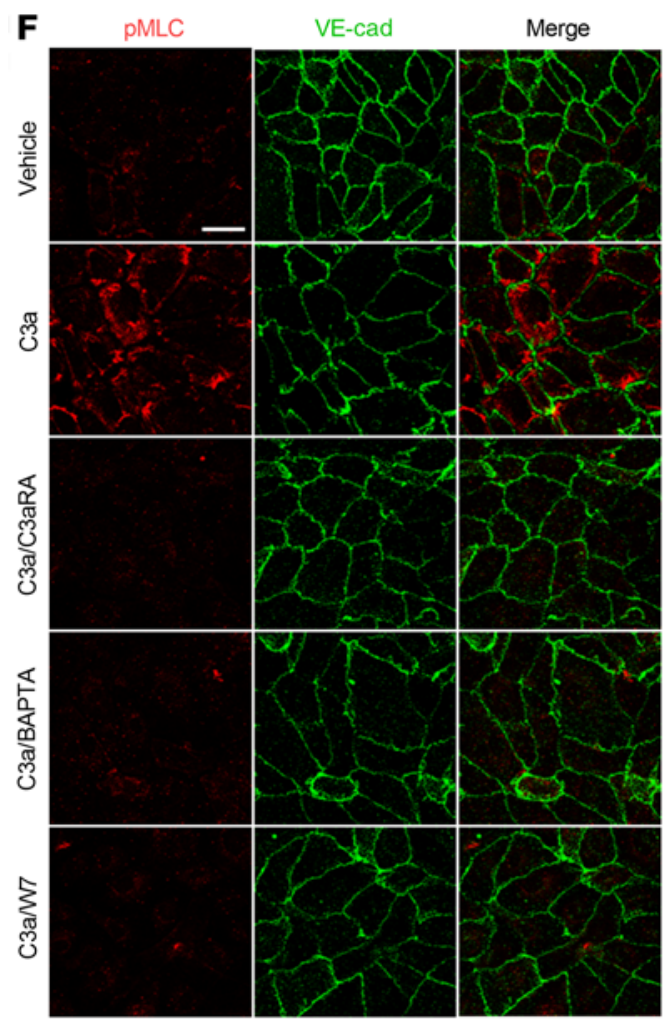

H PMLC VE-cad Merge
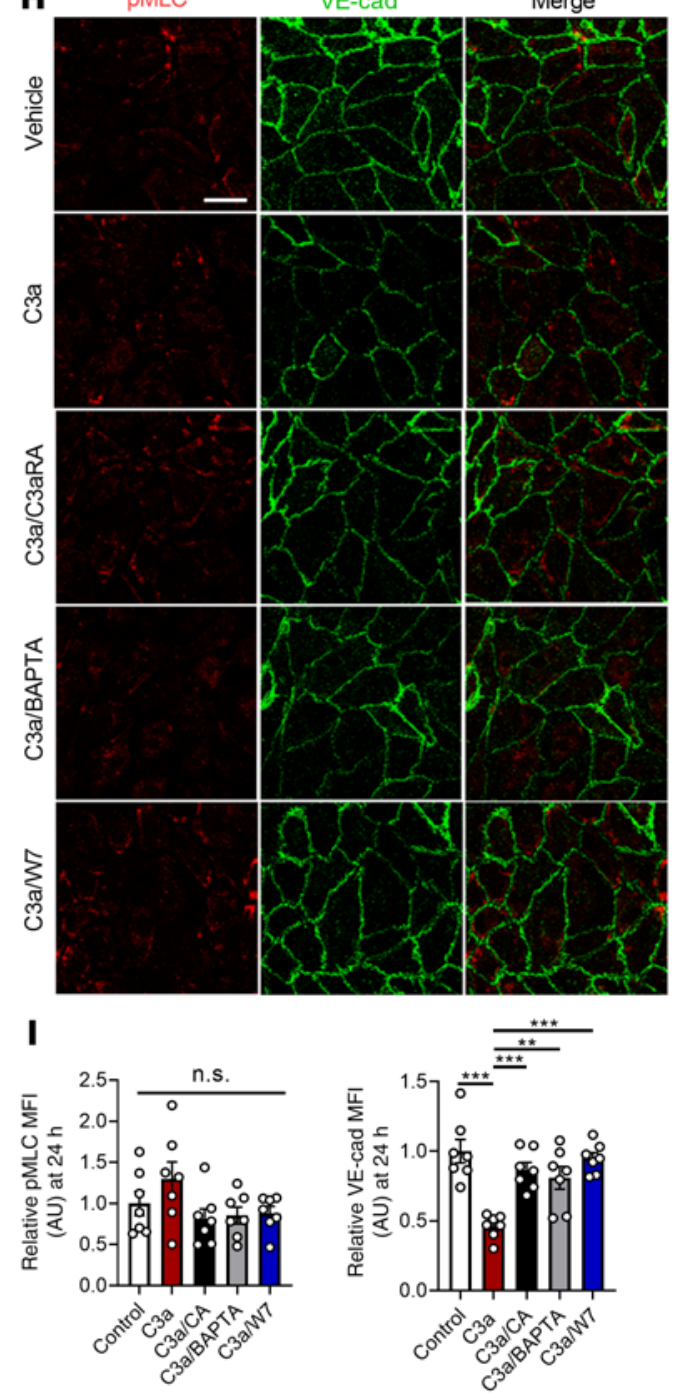
Figure 5. C3a-mediated barrier disruption is dependent on $\mathrm{Ca}^{2+}$ mobilization and alters VE-cadherin through cytoskeletal activation. (A) Schematic of TEER analysis using a coculture of astrocytes and endothelial cells. (B) TEER values in cocultures treated with vehicle, C3a, C3a with C3aRA, or IL-1 $\beta$ for $0,1,4,12$, and 24 hours. (C) Quantification of percentage reduction of TEER at 24 hours from treatments recorded in B. (D) TEER values in cocultures treated with vehicle, C3a, or C3a with C3aRA or BAPTA-AM over 24 hours. (E) Quantification of the percentage of reduction in TEER at 24 hours from the treatments in D. All TEER experiments were performed 2 times with duplicates and normalized to time-point control wells of cell-free membranes. (F) Representative immunofluorescence images of human brain microvascular endothelial cells (HBMECs) treated with vehicle, C3a, or a combination of C3a plus C3aRA (C3a/C3aRA), BAPTA-AM (C3a/BAPTA), or W7 (C3a/W7) for 2 hours and stained with anti-pMLC and anti-VE-cadherin antibodies. (G) Quantification of pMLC or VE-cadherin MFI showed an increase in PMLC but not VE-cadherin ( $n=7$ areas from 3 replicates of $250-300$ cells/condition). (H) Representative immunofluorescence images of HBMECs treated as stated in F using anti-pMLC and anti-VE-cadherin antibodies 24 hours after treatment. (K) Quantification of pMLC or VE-cadherin MFI showed normalized pMLC levels but decreased VE-cadherin ( $n=7$ areas from 3 replicates of 250-300 cells/ condition). All data represent the mean \pm SEM. Analysis was performed on average percentage decrease in TEER using 1-way ANOVA with Tukey's post hoc test $\left({ }^{*} P<0.05,{ }^{* *} P<0.01,{ }^{* *} P<0.001\right)$. Scale bar: $10 \mu \mathrm{m}$.

able to phosphorylate MLC motor protein at Ser19 (pMLC), resulting in stress fiber formation (38). Activation of this pathway is known to initiate a physical, tensile stress at the cell membrane, disrupting VE-cadherin ${ }^{+}$junctions and BBB integrity (39). Therefore, we hypothesized that $\mathrm{C} 3 \mathrm{a}$ might trigger the calcium release needed to disrupt VE-cadherin ${ }^{+}$junctions, resulting in BBB permeability.

To test this hypothesis, we treated endothelial cell monolayers with C3a alone or together with C3aRA, BAPTA-AM, or calmodulin inhibitor W7. Immunofluorescence staining of cells treated with C3a alone for 2 hours showed a robust increase in both phalloidin ${ }^{+}$ F-actin stress fibers and overlapping pMLC signal (Supplemental Figure 9A). To quantify the dynamics of this cellular response, we analyzed changes at 2 hours, during the downward slope of barrier integrity, as measured by TEER analysis, after C3a stimulation. We saw a robust increase in pMLC by both immunofluorescence (Figure 5, F and G) and immunoblotting (Supplemental Figure 9, B and C), both of which were blocked by C3aRA, BAPTA, or W7 treatment. When analyzing the effect on VE-cadherin, we did not see overt changes by either immunofluorescence (Figure 5, F and G) or by immunoblotting (Supplemental Figure 9, B and D) under all conditions, suggesting that $\mathrm{F}$-actin stress fiber formation and activation of pMLC, but not VE-cadherin protein alteration, was involved in the early phase of C3a/C3aR signaling. However, after 24 hours of C3a treatment, pMLC levels normalized, but VE-cadherin levels were significantly reduced by both immunofluorescence staining (Figure 5, H and I) and by immunoblotting (Supplemental Figure 9, E and G). Cotreatment with C3aRA, BAPTA, or W7 normalized VE-cadherin without affecting pMLC (Figure 5I and Supplemental Figure 9, E-G).

Overall, these data established intracellular $\mathrm{Ca}^{2+}$ as a second messenger downstream of $\mathrm{C} 3 \mathrm{a} / \mathrm{C} 3 \mathrm{aR}$ signaling to mediate $\mathrm{pMLC}$ activity and VE-cadherin homeostasis in endothelial cells. These findings suggest that there are 2 phases in endothelial response to C3a. First, a transient phase (2 hours) where endothelial cells respond quickly and rapidly to $\mathrm{C} 3 \mathrm{a}$ signaling by forming stress fibers, followed by a failed ability to maintain VE-cadherin protein levels, leading to barrier permeability (24 hours).

Endothelial cell-specific deletion of C3ar1 rescues vascular phenotypes, reduces microglial reactivity, and corrects age-related neurodegeneration. Because C3ar1 genetic ablation and C3aR pharmacological inhibition were able to rescue age-related changes in brain vasculature, we hypothesized that specific endothelial ablation would show similar effects as global targeting, and that such a manipulation would influence age-related neuroinflammation overall. The above in vitro studies support a role of endothelial C3aR in mediating the barrier permeability. To test this hypothesis directly, we produced mice with conditional deletion of C3ar1 in endothelial cells by crossing a C3ar1-floxed allele (40) with the Tie2-Cre (41) mice to generate C3ar ${ }^{\text {If/l }}$ Tie2-Cre (T2KO) mice. Littermate $\mathrm{C}_{\mathrm{arr}}{ }^{+/+}$and $\mathrm{C} 3 \mathrm{ar} 1^{f / f l}$ mice were used as controls. The cell type-specific knockout was confirmed by immunofluorescent imaging (Supplemental Figure 10A).

Coimmunofluorescent staining of control and $\mathrm{T} 2 \mathrm{KO}$ mice at 3 and 12-14 months of age with anti-VCAM1 and anti-CD31 antibodies revealed significant increases in cortical vascular VCAM1 signal at 12-14 months of age in the control group (Figure 6A). Similar to the germline deletion, age-associated elevation of VCAM1 expression was almost completely attenuated in the T2KO mice (Figure 6, A and B), which was also seen in the hippocampus (Supplemental Figure 10, B and C). Analysis of vessel morphology by CD31 staining and 3D reconstruction showed a significant reduction in vessel cross-sectional area in 12-14-month-old control mice compared with that of 3-month-old mice (Figure 6, C and D). Consistent with the VCAM1 staining, endothelial deletion of C3ar1 was sufficient to rescue the age-associated changes of vessel morphology (Figure 6, C and D). These data demonstrated that specifically ablating $\mathrm{C} 3 \mathrm{ar} 1$ in endothelial cells rescued agerelated changes in brain vasculature, similar to global ablation and pharmacological inactivation. Thus, endothelial C3aR played a cell-autonomous role in mediating age-dependent changes in vascular inflammation and morphology.

It was previously reported that reducing VCAM1 expression in endothelial cells can benefit brain function (11). Thus, we tested whether inhibiting the C3aR/VCAM1 axis at the endothelial cells could influence microglial reactivity. Coimmunostaining of 2- and 12-month-old WT and C3ar1 ${ }^{--}$mice with microglia marker IBA1 and a marker for phagocytic microglia, CD68, followed by colocalization analysis identified a higher percentage of CD68 signal colocalized with IBA1 in 12-month-old WT mice, which was completely normalized by global C3aR inactivation (Figure 7, A and B). Analysis of T2KO mice and their littermate controls at 12-14 months showed a partial but significant reduction of CD68 immunoreactivity (Figure 7, C and D). This result suggests that although C3aR is expressed in other cell types, notably microglia, it also plays a role in mediating neuroinflammation in the brain by modulating the endothelial C3aR/VCAM1 axis and promoting peripheral immune cell interaction at the brain vasculature.

To determine whether the changes of immune cells detected in the middle age (12-14 months) may lead to neuronal loss later in life, we performed Nissl staining of brain sections from young (3 months) and old (20 months) WT control mice and 20-month-old ${\mathrm{C} 3 \mathrm{arl}^{-/}}^{-}$and T2KO mice (Supplemental Figure 11) and quantified 
A
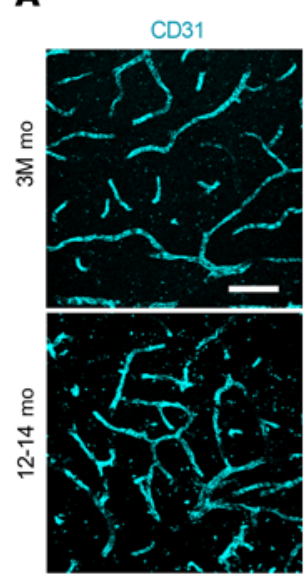

C
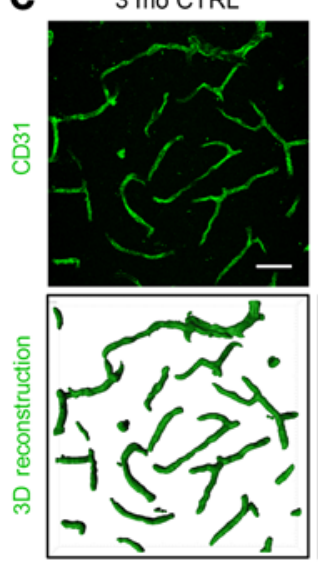

CTRL
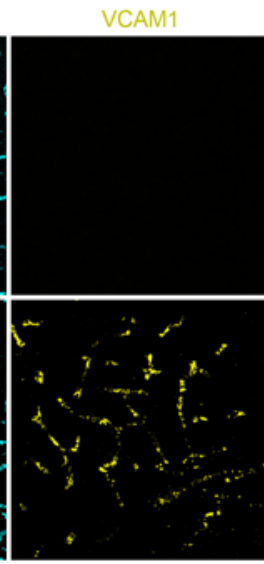

$3 \mathrm{mo} \mathrm{T} 2 \mathrm{KO}$
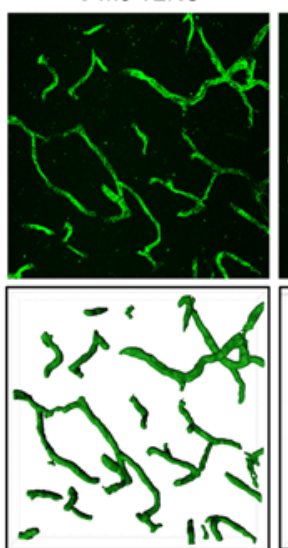

12-14 mo CTRL
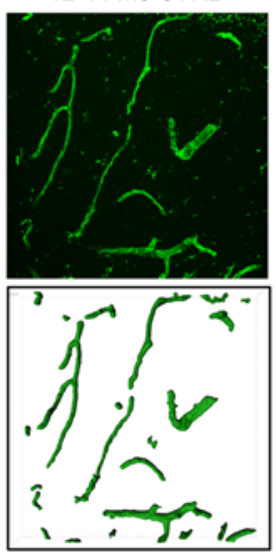

12-14 mo T2KO
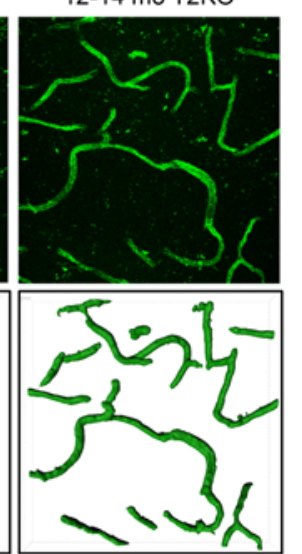

CD31
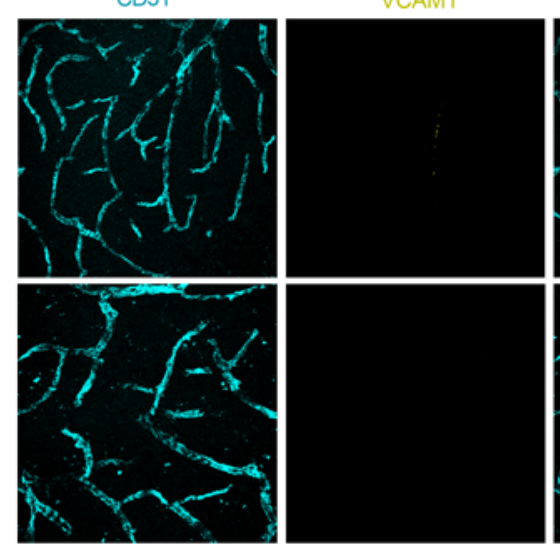

B
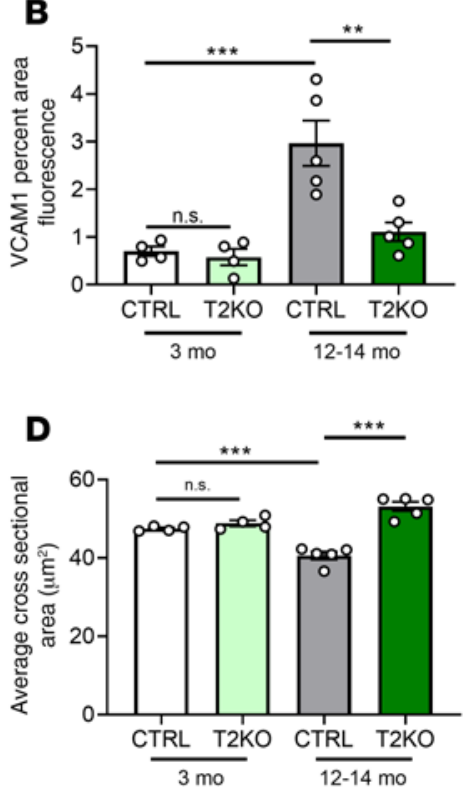

Figure 6. Conditional knockout of C3ar1 in brain endothelial cells rescues age-related vascular phenotypes. (A) Representative VCAM1 and CD31 double-staining images from 3-month-old and 12- to 14-month-old endothelial C3ar1 conditional knockout (T2KO) mice and littermate controls (CTRL) showing increased VCAM1 expression with age in CTRL mice but suppressed expression in T2KO. (B) Quantification of VCAM1 intensity of A. (C) Representative CD31 staining and $3 D$ reconstruction of 3-month-old and 12- to 14-month-old CTRL and T2KO mice. (D) Quantification of average CD31+ cross-sectional areas. All data represent the mean \pm SEM of $n=4$ /group. Analysis for A-F was performed using 1-way ANOVA with Tukey's post hoc test ( ${ }^{* *} P<0.01$, $\left.{ }^{* *} P<0.001\right)$. Scale bar: $50 \mu \mathrm{m}$.

hippocampal and piriform/entorhinal cortical volumes (Figure 7, $\mathrm{E}$ and $\mathrm{F}$ ). We found a mild but significant reduction in tissue volumes with age in the control animals. Interestingly, global and endothelial cell-specific ablation of C3aR led to a comparable degree of rescue (Figure 7, E and F). Together, these data suggest that blocking the endothelial C3aR/VCAM1 axis and restoring endothelial vascular morphology could restore microglial function and restore brain health during aging.

C3aR modulates vascular changes in PS19 tau-transgenic mice. Our previous analysis of the widely used PS19 tau-transgenic mouse model reported heightened C3/C3aR signaling associated with hyperphosphorylated tau pathology, such that genetic deletion of C3ar1 effectively reduced tau pathology and neuroinflammation (22). Examination of the previously reported brain transcriptomes of PS19 and PS19 C3ar1 ${ }^{-/-}$mice (22) clearly demonstrated the capacity for $\mathrm{C} 3 \mathrm{aR}$ to modulate the innate immune response. Further, overrepresentation analysis of this gene expression data found KEGG and Reactome pathways upregulated in PS19 and downregulated in PS19 $\mathrm{C}_{3} \mathrm{arl}^{-/-}$, consistent with cytokine activation, leukocyte activation and migration, cell adhesion molecule interactions, and regulation or activation of the cytoskeleton, all of which are consistent with endothelial responses to C3a (Figure 8A). In addition to leukocyte transcripts in our pathway analysis, specific transcripts identifying peripheral immune cell infiltration (Vcam1, Ptpn22, Cd3e, and Cd8a) were also significantly elevated in PS19 brains (Figure 8A and Supplemental Figure 12).

Given the changes in endothelial processes, in addition to the peripheral immune cell response, we hypothesized that the elevated C3a/C3aR/VCAM1 pathway contributes to vascular changes in PS19 mice. Indeed, coimmunofluorescent analysis of cortical vasculature of 9-month-old PS19 mice labeled with CD31 and VCAM1 revealed a significant increase in VCAM1 expression colocalized with $\mathrm{CD} 31^{+}$vasculature, and this phenotype was rescued to control levels by ablating C3ar1 (Figure 8, B 
A
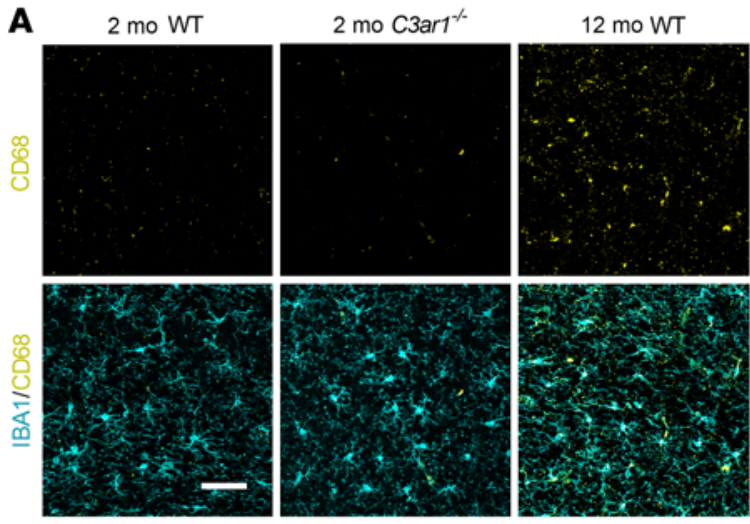
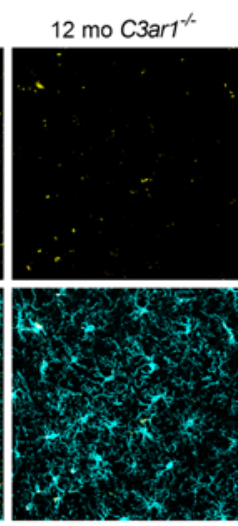
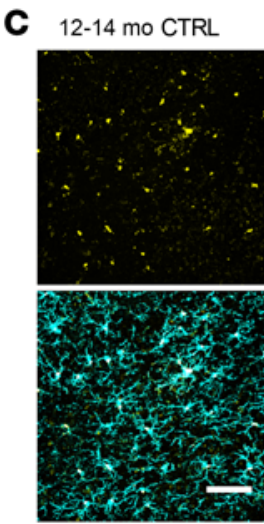

$12-14 \mathrm{M}$ T2KO
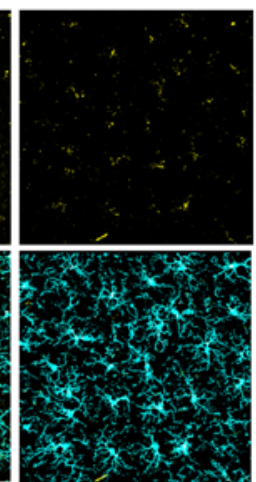

B

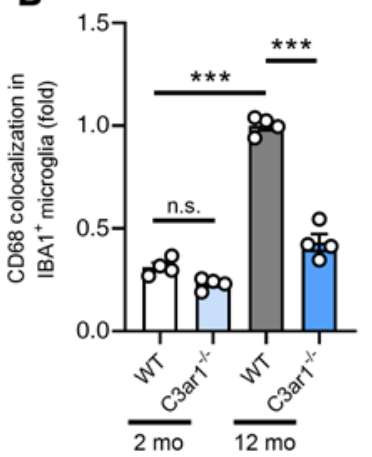

D

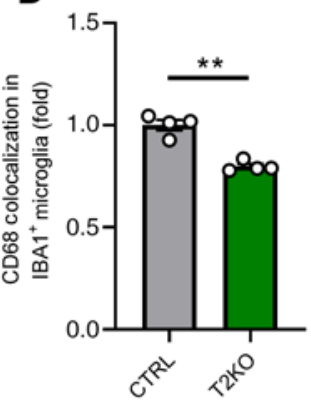

$\mathbf{E}$

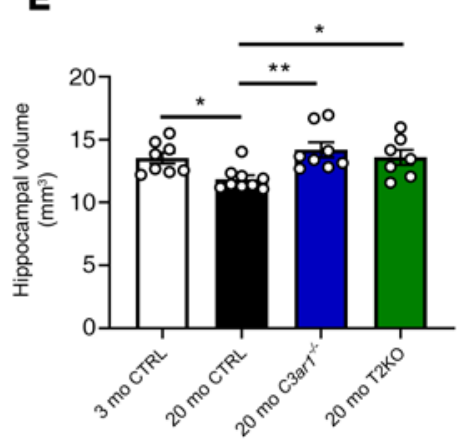

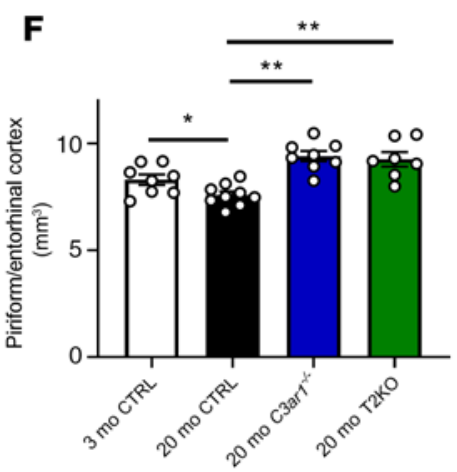

Figure 7. Germline and conditional knockout of C3ar1 rescues age-related microglial reactivity and neurodegenerative phenotypes. (A) Representative IBA1

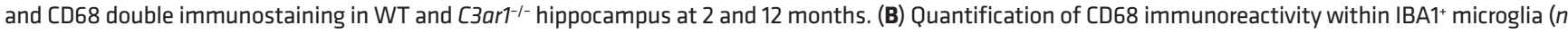
= 4/group). (C) Representative IBA1 and CD68 double immunostaining in CTRL and T2KO hippocampus at 3 months and 12-14 months. (D) Quantification of CD68 immunoreactivity within IBA1 ${ }^{+}$microglia ( $n=4$ /group). (E) Quantification of hippocampal volume through coronal, serially sectioned tissue samples ( $n=$ 7-9/group, 9 sections/animal quantified). (F) Quantification of entorhinal cortex volume through coronal, serially sectioned tissue samples ( $n=7-9 / g r o u p, ~ 9$ sections/animal quantified). Data for $\mathbf{B}$ and $\mathbf{E}$ represent the mean $\pm \mathrm{SEM}$, and analysis was performed using 1-way ANOVA with Tukey's post hoc test ( ${ }^{*} P<0.05$, ${ }^{* *} P<0.01$, $\left.{ }^{* * *} P<0.001\right)$. Data for $\mathbf{D}$ represent the mean \pm SEM, and analysis was performed using a 2-tailed Student's $t$ test $\left({ }^{* *} P<0.01\right)$. Data for $\mathbf{F}$ represent the mean \pm SEM and analysis was performed using 1-way ANOVA with Holm-Sidak post hoc test $\left({ }^{*} P<0.05,{ }^{* *} P<0.01\right)$. Scale bar: $50 \mu \mathrm{m}$ (A and C).

and C). Increased VCAM1 expression in PS19 mice was accompanied by drastic reduction in the average cross-sectional area of cortical brain vasculature (Figure 8, D and E). Consistent with aging analysis, C3ar1 ablation in PS19 animals significantly improved vascular morphology (Figure 8, D and E). These data revealed a vascular phenotype associated with tau pathology and suggest that the endothelial C3aR/VCAM1 axis contributes to vascular dysfunction in tauopathy.

\section{Discussion}

Our data provided evidence that activated C3a/C3aR signaling in brain endothelial cells triggered an increase in the cell adhesion molecule VCAM1 and initiated an inflammatory transition affecting brain vascular structure and function during aging. This was associated with lymphocyte infiltration, altered vascular morphology, increased BBB permeability, and, ultimately, age-related neurodegeneration. Genetic or pharmacological $\mathrm{C} 3 \mathrm{aR}$ inhibition rescued these age-associated phenotypes. Our in vitro $\mathrm{BBB}$ model implicates endothelial C3aR in the barrier disruption and suggests downstream intracellular $\mathrm{Ca}^{2+}$ signaling and VE-cadherin localization and expression as the underlying mechanisms. A cell-autonomous effect of endothelial C3aR was further validated by our demonstration that endothelial cell-specific ablation of C3arl phenocopied germline deletion with regard to vascular phenotypes. Analysis of the endothelial cell-specific C3ar1-knockout mice also supports the notion that morphological and functional changes in vasculature contribute to microglial reactivity and age-related neurodegeneration. Finally, we documented that similar vascular phenotypes and their C3aR dependency were observed in PS19 tau-transgenic mice, supporting a common C3a/C3aR/VCAM1 signaling pathway in the mediation of neuroinflammation in aging and neurodegenerative disease.

To determine the functional consequence of the activated endothelial C3a/C3aR axis during aging, we analyzed VCAM1 expression and peripheral immune cell infiltration in the brain and found a substantial increase in $\mathrm{CD}^{+} \mathrm{T}$ cells, whereas genetic and pharmacological inhibition of the C3a/C3aR pathway reduced these phenotypes. This likely occurred through a VCAM1-regulated process, because the interaction between vascular VCAM1 and its lymphocyte-expressed ligand VLA-4 is well established (42). These findings point to a strong shift toward vascular inflammation in brain endothelial cells during aging.

A recent report by Yousef et al. concluded that elevated brain VCAM1 expression activated microglia and decreased neural stem 
A

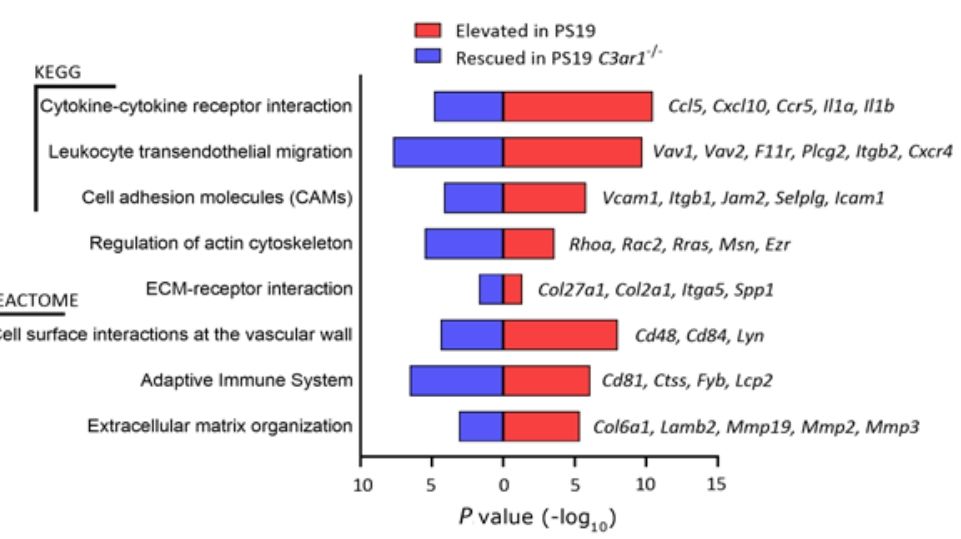

B

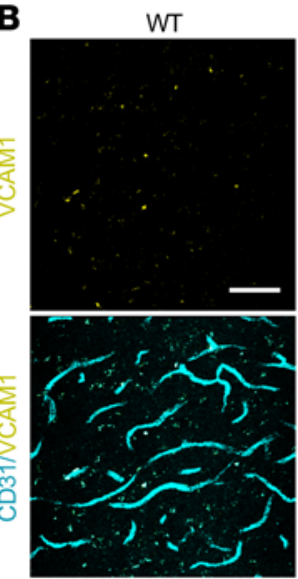

D
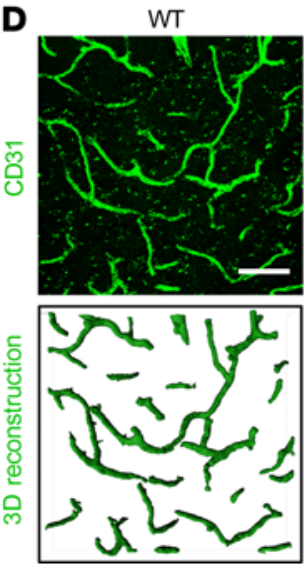

C3ar1
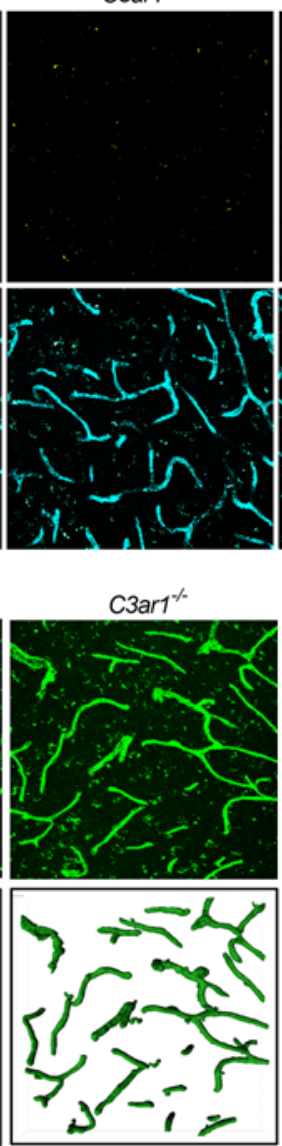

PS19

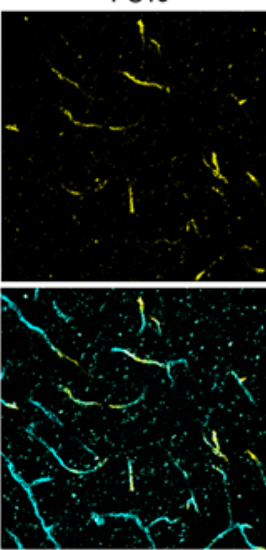

PS19
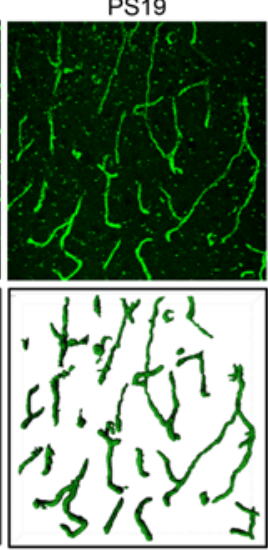

PS19 C3ar1\%

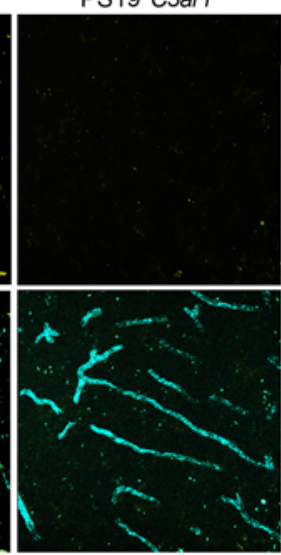

PS19 C3ar1\%

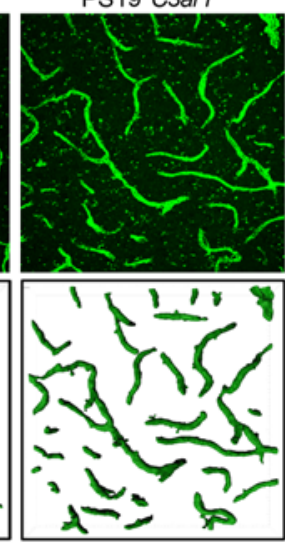

C

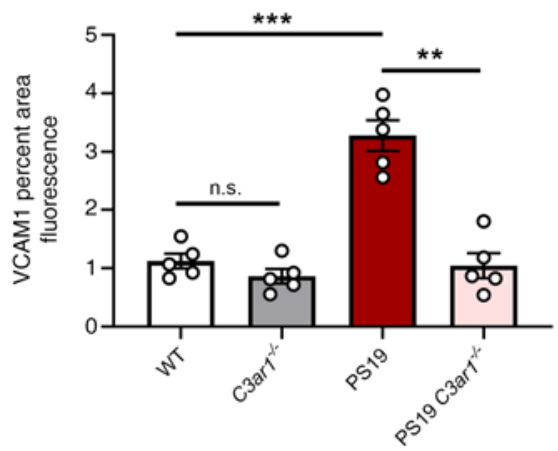

E

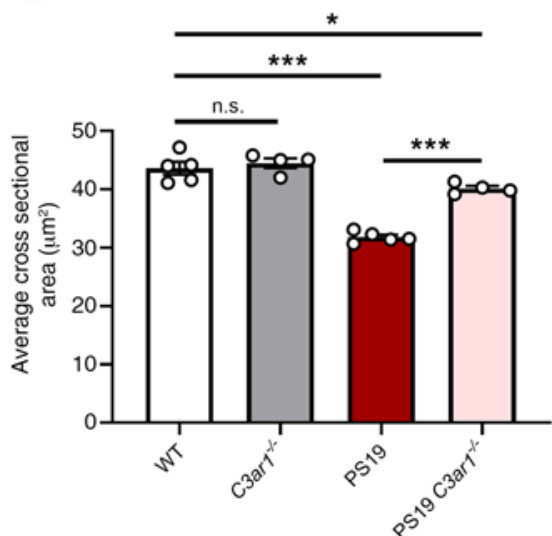

Figure 8. Vascular abnormalities in PS19 tau-transgenic mice and C3aR dependency. (A) RNA-Seq analysis revealed significantly overrepresented pathways in the differentially expressed genes (DEGs) that were increased in 9-month-old PS19 compared with WT animals (red), and that were decreased in PS19 C3ar1/compared with PS19 animals (blue). Terms were selected from results based on their involvement in vascular biology and immune cell infiltration and plotted by $P$ value; representative rescued genes contributing to the terms are listed (right). (B) Cortical staining of 9-month-old WT, C3ar1 ${ }^{-/-}$, PS19, and PS19 C3ar1 ${ }^{-/-}$hippocampal vasculature with CD31 and VCAM1 demonstrated a significant increase in VCAM1 expression in PS19 mice and a rescue of this phenotype in PS19 mice harboring the C3ar1 deletion. (C) Quantification of VCAM1 immuno-intensity. (D) CD31 staining and IMARIS-aided 3D reconstruction of 9-month-old WT, C3ar1 ${ }^{-/-}$, PS19, and PS19 C3ar1 ${ }^{-1-}$ hippocampal vasculature. (E) Quantification of the average vessel cross-sectional area. All data represent the mean \pm SEM of $n=5 /$ group. Analysis for all results was performed using 1-way ANOVA with Tukey's post hoc test $\left({ }^{*} P<0.05,{ }^{* *} P<0.01,{ }^{* * *} P<0.001\right)$. Scale bar: $50 \mu \mathrm{m}$.

cell numbers in aged mice (11). They also addressed the peripheral immune cell contribution by using $\alpha$ VLA-4-blocking antibodies in 16-month-old mice, showing a rescue of these phenotypes. Work by Dulken et al. also suggested that increased prevalence of clonally expanded $\mathrm{CD}^{+} \mathrm{T}$ cells in the brains of 28- to 29-month-old mice significantly hindered neural stem cell fitness in the subventricular zone (13). These findings, along with ours, support a model of periodic immune cell infiltration and eventual clonal expansion during aging. Our C3aR inhibition studies and the VLA-4 blockade used by Yousef et al. suggest that blocking peripheral immune 
cell recruitment during aging may reduce microglial reactivity and dampen the activated neuroimmune environment, whereas allowing this to go unchecked in old mice results in further $\mathrm{CD}^{+} \mathrm{T}$ cell infiltration and clonal expansion (of both early and late recruits). The partial rescue of infiltration in our 20-month-old pharmacological inhibition model supports this supposition of periodic, age-dependent waves of infiltration. Although our study did not address neural stem cell fitness or fate, we showed a C3aR-dependent effect on age-related neurodegeneration in our C3ar1-null and T2KO mouse models. Together, the current findings suggest that, in part, age-related neurodegeneration might be provoked by peripheral immune cell interaction with or signaling to microglia. Future studies should analyze more time points specifically in a later stage of life to determine the exact windows of infiltration that affect brain function and to clearly identify the role of these $\mathrm{CD}^{+} \mathrm{T}$ cell infiltrates. Recent work by Kolev et al. showed that peripheral immune cells elevate intrinsic C3 production after diapedesis into peripheral tissues (43). They suggest a prominent role for the $\mathrm{CD}^{+} \mathrm{T}$ cell interaction (via LFA-1) with endothelia (via ICAM1), but also identify a similar effect in $\mathrm{CD}^{+} \mathrm{T}$ cells stimulated with VCAM1, showing intrinsic upregulation of IFN- $\gamma$ and C3 (43). Further dissection of this mechanism with respect to $\mathrm{CD}^{+} \mathrm{T}$ cells and VCAM1 could shed light on potential feedforward mechanisms affecting microglial reactivity during aging.

We also analyzed structural and morphological characteristics of brain vasculature in the hippocampus, because this region was previously reported to undergo age-related vascular dysfunction in the form of BBB disruption and reduced cerebral blood flow (44, 45 ). We found that $\mathrm{C} 3 \mathrm{a} / \mathrm{C} 3 \mathrm{aR}$ signaling induced structural changes in vasculature affecting the vessel cross-sectional area and vessel tortuosity, characteristics previously associated with impaired cerebral blood flow and abnormal angiogenesis (34). Additional work is needed to understand the exact impact of C3a on hemodynamics. Work by the Zlokovic group has shown that the BBB undergoes age-related permeability in the hippocampus prior to disease $(10,44-46)$. We found that aged mouse brain vessels were more permeable to a BBB-impenetrable tracer dye and confirmed the loss of barrier integrity by analyzing FACS-isolated endothelial cells, which showed impaired gene expression of critical BBB genes (Cdh5, Ocln, Tjp1, and Cldn5). Our data showed that C3a/ $\mathrm{C} 3 \mathrm{aR}$ signaling was partly responsible for these structural and functional changes in BBB integrity during aging.

Our data using dextran indicated that the immune cell infiltration by acute LPS treatment was an active process, rather than the result of a compromised $\mathrm{BBB}$. This interpretation differs from a previous report using heavy isotope- and radio-labeled proteins, which revealed increased BBB permeability by LPS induction (47). Although the exact cause for this discrepancy is not clear, recent work from the Wyss-Coray laboratory identified potentially novel, agerelated, receptor-mediated transcytosis mechanisms for protein transfer across the BBB (48). It is possible that a similar transcytosis mechanism could underlie the presence of heavy isotope- or radio-labeled proteins in the brain in acute neuroinflammatory models. Given this new development, further work should be done to better understand BBB permeability in acute neuroinflammation.

Mechanistic analysis of the endothelial barrier phenotype suggests a role for calcium-mediated signaling in an in vitro model of the BBB. Further analysis identified a potential phase response, in which initial calcium-mediated signaling induced phosphorylation of MLC protein, resulting in VE-cadherin protein loss at intercellular junctions. These effects were rescued by inhibition of $\mathrm{C} 3 \mathrm{aR}$ or calmodulin in endothelial cell cultures, establishing a strong link between $\mathrm{C} 3 \mathrm{a} / \mathrm{C} 3 \mathrm{aR}$ activation and calcium-mediated effects in brain endothelial cells. Together, these findings showed that changes in brain vessel structure, hemodynamics, and BBB permeability may be directly modulated by glial reactivity and other complement-related changes seen in aged brains.

The major focus of this study addresses the role of agerelated vascular changes due to $\mathrm{C} 3 \mathrm{a} / \mathrm{C} 3 \mathrm{aR}$ signaling; however, we observed similar activation in the PS19 model of tauopathy. In our previous study of this model (22), we identified a key C3aRdependent microglial activation network and showed that blocking $\mathrm{C} 3 \mathrm{aR}$ corrected microglial activation and other transcriptional changes. Activation of the $\mathrm{C} 3 \mathrm{a} / \mathrm{C} 3 \mathrm{aR}$ network also influenced gene expression signatures consistent with peripheral immune cell activation, a phenomenon previously reported in other Alzheimer disease mouse models $(49,50)$. Given this, we reasoned that impaired vasculature and endothelial cell response may be partly responsible for the presence of peripheral immune cell signatures. Indeed, RNA-Seq analysis revealed increased levels of Vcam1, Cd3e, Cd8a, and Ptpn22 genes in PS19 hippocampi, and histology revealed highly altered vessel morphology. Previous work by Faraco et al. demonstrated a role of hypertension in potentiating accumulation of hyperphosphorylated tau, a finding which corroborates our belief that endothelial structure and function may influence disease pathogenesis (51). The study by Laurent et al. used a $\mathrm{CD}^{+} \mathrm{T}$ cell depletion strategy showing that Clec7a, Itgax, Cd68, and even astrocytic Gfap mRNA levels are reduced by depleting T cells (50). Although our current study does not address the downstream effect of these vascular changes as it relates to disease progression, it does highlight a possible role for endothelial cells in potentiating microglial reactivity through vascular function and peripheral immune cell interactions. Together, these studies position endothelial cells as the gatekeepers to disease progression through the C3a/C3aR/VCAM1 axis.

In conclusion, our work identifies a potentially novel complement regulatory axis at the BBB through endothelial C3aR. It implicates a critical role for a C3aR-dependent endothelial inflammatory transition, which results in increased VCAM1 expression in the aged brain. Our data suggest that blocking complement-mediated effects can have a substantial impact on improving vascular health, rescuing BBB permeability, and decreasing neuroinflammation in aging and neurodegeneration. Since the complement pathway is upregulated in both acute inflammatory conditions, such as stroke and traumatic brain injury, and in neurodegenerative diseases, in particular Alzheimer disease, of which age is the greatest risk factor, our findings have direct implications for the pathogenesis and therapeutic targeting of these age-related diseases of the brain.

\section{Methods}

For additional details, see Supplemental Methods.

Mice and treatment. The aged C57BL/6J mice were obtained from the aging rodent colony of the NIH National Institute on Aging. C3ar1-deficient mice ( $\left.\mathrm{C} \mathrm{arr}^{-/}\right)$mice were obtained from the Jackson 
Laboratory and backcrossed to C57BL/6J for 5 generations. Mice were housed 2-4 per cage in a pathogen-free mouse facility with ad libitum access to food and water on a 12-hour light/12-hour dark cycle. Mice for the conditional knockout studies were bred from a mixed background of Tie2 ${ }^{\text {cre }}$ mice (C57BL/6J) and C3ar1 ${ }^{f /+}$ mice (BALB/c) (40). The breeding scheme for PS19 studies was previously published by Litvinchuk et al (22). Males and females in approximately equal numbers were used for all experiments.

Vehicle (0.5\% DMSO) or C3aR antagonist (C3aRA $1 \mathrm{mg} / \mathrm{kg}$ ) were i.p. injected every other day for 4 weeks. For i.c.v. administration of LPS, mice were placed in a Kopf stereotaxic instrument, and glass needles were inserted through bore holes using coordinates to target lateral ventricles $(-0.4 \mathrm{~mm}$ anteroposterior, $\pm 1.0 \mathrm{~mm}$ mediolateral, and $-2.0 \mathrm{~mm}$ dorsoventral from the surface of the skull at bregma). LPS (2 $\mu \mathrm{g} / \mathrm{mL}$ ) or vehicle (PBS) was administered bilaterally ( $2 \mu \mathrm{L}$ each side).

BBB analysis was followed as previously described (35). Briefly, mice were injected via tail vein with $100 \mu \mathrm{L}$ of $10 \mathrm{mg} / \mathrm{mL}$ stock TRITCdextran (MW 65-85 kDa; Sigma-Aldrich, T1162). Dye was allowed to naturally perfuse for 2 hours and was then perfused with PBS. One hemisphere was used to determine TRITC fluorescence signal in tissue homogenates (excitation $\lambda 550 \mathrm{~nm}$, emission $\lambda 580 \mathrm{~nm}$ ) using a plate reader (Molecular Devices Spectra Max i3x). The other hemisphere was fixed in $4 \%$ PFA overnight at $4^{\circ} \mathrm{C}$ and switched to $30 \%$ sucrose. Sagittal brain sections $(30 \mu \mathrm{m})$ were cut on a sliding microtome, washed in PBS, and stained with lectin-649 (Vector Labs, DL-1178) for 30 minutes at room temperature in PBS containing 0.4\% Triton X-100, $4 \%$ donkey serum, and $1 \%$ BSA to mark brain vasculature. The sections were imaged on a Leica TCS laser confocal microscope at $40 \times$ under oil immersion, with a $Z$-step of $0.5 \mu$ m over a total range of $30 \mu \mathrm{m}$.

Cell culture. Primary HBMECs were obtained from Cell Systems (ACBRI 376). Cells were thawed and plated into T75 flasks for expansion in Lonza EGM2-MV medium (CC-3202) to reach a P4 culture. Cells were subcultured until confluent, passaged at a 1:4 ratio into T75 flasks for P5 cultures, and allowed to expand until confluent prior to freezing (EGM2-MV plus 10\% DMSO). Fresh vials were thawed to obtain P6 cultures, which were used for all further experiments.

Primary human astrocytes were obtained from ScienCell Research Laboratories (ScienCell, 1800). Cells were thawed and plated into a T75 flask for expansion in astrocyte medium (AM) (ScienCell, 1801). Cells were subcultured until near confluence (80\%90\%), passaged at a 1:4 ratio into T75 flasks, and allowed to expand until near confluence prior to freezing (AM plus 10\% DMSO). Fresh vials were thawed and used for all further experiments.

Primary mouse astrocyte cultures were prepared as described previously (20) and purified using negative selection by magnetic CD11b beads (Miltenyi Biotec, 130-049-601). Primary HBMECs were cultured on $100 \mu \mathrm{g} / \mathrm{mL}$ fibronectin and Col IV-coated 24-well or 48-well plates. Cells were seeded at a density of $2.5 \times 10^{5}$ cells $/ \mathrm{cm}^{2}$. Confluent cells were treated with IL-1 $(10 \mathrm{ng} / \mathrm{mL}, \mathrm{R} \& \mathrm{D}$ Systems, 201-LB-005), C3a (500 nM, R\&D Systems, 3677-C3-025), C5a (250 nM, R\&D Systems, 2037-C5-025), ionomycin (10 $\mu \mathrm{M}$, Cayman Chemical, 10004974), or in combination with one of the inhibitors SB290157 (5 $\mu \mathrm{M}$, Calbiochem, 559410), W7 (50 $\mu \mathrm{M}$, Tocris, 0369) or BAPTA-AM (1 $\mu \mathrm{M}$, Tocris, 2787). Cells were analyzed after 2 hours or 24 hours of treatment.

TEER analysis was performed using combinations of primary HBMECs and primary human or mouse astrocytes in a coculture. Briefly, semipermeable transwell inserts (Corning, 3470) were coated with
$100 \mu \mathrm{g} / \mathrm{mL}$ fibronectin and Col IV on the luminal surface for 2 hours at $37^{\circ} \mathrm{C}$ in PBS. The remaining coating solution was aspirated, and the transwells were flipped over and placed into 12 -well culture plates. The abluminal surface was coated with poly-D-lysine (PDL) at $37^{\circ} \mathrm{C}$ for 2 hours, and the remaining solution was aspirated. While inverted, primary astrocytes were seeded to the abluminal surface at a density of $1.5 \times 10^{5}$ cells $/ \mathrm{cm}^{2}$ and allowed to attach for $4-6$ hours at $37^{\circ} \mathrm{C}$ in $100 \mu \mathrm{L} \mathrm{AM}$ (ScienCell). The membranes were then reverted to normal position in their original culture plate, and the astrocytes were cultured in AM placed into the tissue culture plate (abluminal). The cells were cultured for 48 hours at $37^{\circ} \mathrm{C}$ and endothelial cells were seeded in the luminal compartment at a density of $1.5 \times 10^{5} \mathrm{cells} / \mathrm{cm}^{2}$ in EGM2-MV. All TEER readings were measured using STX2 chopstick electrodes with an EVOM2 volt/ ohm meter (World Precision Instruments). Cultures matured over 3-4 days, and when TEER stabilized ( $160-180 \Omega$ ), treatments were added and TEER was monitored over 24 hours. All TEER readings were normalized to the average reading from 2 cell-free inserts for each timepoint recording prior to normalization to the control samples.

Brain vessel preparations. Isolation of mouse brain vessels was carried out as previously described (28) with minor modifications. Briefly, mice were perfused with PBS, brains were removed, and cerebellum olfactory bulb and brain stem were discarded. They were stripped of dura and meninges, gently sliced with a razor blade, and gently homogenized using a glass Dounce homogenizer (Kontes Glass, 19), all on ice. The homogenate was centrifuged, supernatant was discarded, and the pellet was resuspended in a dextran solution to remove myelin debris. The resulting pellet was then filtered over a $40 \mu \mathrm{m}$ filter and vessel fragments were retained in the filter. The filter was then turned over, placed on a $50 \mathrm{~mL}$ conical tube, and rinsed. Vessel fragments were pelleted at $300 \mathrm{~g}$ for 5 minutes and fixed in $4 \%$ PFA for 30 minutes on ice. Fixed fragments were pelleted at $300 \mathrm{~g}$ for 5 minutes, washed with PBS, and mounted onto manually gridded slides for staining. Vessels were blocked with PBS containing 0.4\% Triton X-100, 4\% donkey serum, and 1\% BSA for 30 minutes, and incubated in blocking solution with primary antibody overnight at $4^{\circ} \mathrm{C}$. Depending on the experiment, primary antibodies were used as follows: rabbit antiGFAP (MilliporeSigma, G9269), rat anti-C3aR (Hycult, 10130173), and goat anti-mVE-cadherin (R\&D Systems, AF1002). Imaging was performed on a Leica TCS laser confocal microscope at $63 \times$ under oil immersion, with a $Z$-step of $0.5 \mu \mathrm{m}$ over a total range of $10 \mu \mathrm{m}$.

Flow cytometry analysis. Flow cytometry analysis of aged brain lymphocytes was performed using CoBrA dissociation strategy as previously described (27), with slight modifications for myelin/debris removal, antibody staining, and for dissociation to subtype lymphocyte markers. Briefly, adult mice were perfused with PBS, brain tissues were gently minced with sterile razor blades, digested in papain (Worthington Biochemical, LK003172) and DNase (Worthington Biochemical, LK003178), and then triturated 3-4 times using a fire-polished glass Pasteur pipette. After incubation, papain digestion was neutralized with HBSS+ and the suspension was pelleted at $310 g$ for 5 minutes at $4^{\circ} \mathrm{C}$. The pellet was resuspended in $1 \mathrm{~mL}$ of HBSS+, transferred to an ice-cold $1.7 \mathrm{~mL}$ Eppendorf tube and further triturated 3 times, and the supernatant was collected after a brief, low-speed centrifugation. The supernatant at the end of each brief centrifugation was filtered through a prewetted $40 \mu \mathrm{m}$ cell strainer (BD Biosciences, 352340) into a chilled $50-\mathrm{mL}$ conical tube and centrifuged at $310 g$ for 5 minutes at $4^{\circ} \mathrm{C}$. The resulting pellet was depleted of myelin and other debris using a $20 \%$ 
isotonic Percoll PLUS (MilliporeSigma, E0414-250ML) separation. The resulting pellet contained dissociated single cells. For myeloid versus lymphoid discrimination, cells were incubated in $500 \mu \mathrm{L}$ HBSS+ containing 1:100 Mouse BD Fc Block (BD Biosciences, 553141), 1:500 rat anti-CD45-BV421 (BD Biosciences, 563890), and 1:500 rat antiCD11b-FITC (BD Biosciences, 553310) on ice for 15-20 minutes. For subtyping the lymphocyte populations, the tissue dissociation strategy was changed using Collagenase/Dispase (MilliporeSigma, 10269638001) in place of papain to preserve the epitopes for CD19, CD8a, and CD4. All other steps of the dissociation strategy remained the same. After tissue dissociation, cells were incubated in 1:500 rat anti-CD45-BUV395 (BD Biosciences, 564279), 1:500 rat anti-CD11bFITC (BD Biosciences, 553310), hamster anti-CD3e-BV650 (BD Biosciences, 564378), 1:500 rat anti-CD19-BV480 (BD Biosciences, 566167), 1:500 rat anti-CD4-PE (BD Biosciences, 553730), and 1:500 rat anti-CD8a-APC (BD, 553035) on ice for 15-20 minutes. Cells were washed twice with HBSS+ and resuspended in $500 \mu \mathrm{L}$ of HBSS+ prior to flow cytometry analysis. Flow analysis was performed using a BD Biosciences LSR Fortessa equipped with 355 nm, 405 nm, 488 nm, 561 $\mathrm{nm}$, and $640 \mathrm{~nm}$ lasers to minimize spectral overlap.

For FACS of astrocytes and endothelial cells, see the tissue preparation methods using papain. After tissue dissociation, cells were incubated in $500 \mu \mathrm{L}$ HBSS+ containing 1:100 Mouse BD Fc Block (BD Biosciences, 553141), LIVE/DEAD Fixable Blue Dead Cell (Thermo Fisher Scientific, L23105), 1:500 rat anti-CD45-BV421 (BD Biosciences, 563890), and 1:500 rat anti-CD11b-FITC (BD Biosciences, 553310), 1:250 anti-CD49a-VioBright PE (Miltenyi Biotec, 130-107-632), and 1:100 anti-ACSA-2-APC (Miltenyi Biotec, 130-116-245) on ice for 15-20 minutes. Endothelial cells were sorted by first excluding $\mathrm{CD} 45^{+}$and $\mathrm{CD}_{11} \mathrm{~b}^{+}$cells and gating around CD $49 \mathrm{a}^{+}$cells. Astrocytes were sorted by gating triple negativity for the former markers and finally gating around $\mathrm{ACSA}^{+}$cells. Sorting was performed using a BD Biosciences Aria II on the $100 \mu \mathrm{m}$ nozzle. Cells were sorted into $1.7 \mathrm{~mL}$ Eppendorf tubes containing $200 \mu \mathrm{L}$ HBSS+, followed by centrifugation and lysis of pellets in Qiagen RLT buffer containing $1 \% \beta$-mercaptoethanol.

For flow cytometry of HBMECs, cells were singularized with trypsin EDTA (Thermo Fisher Scientific, 25200056) for 5 minutes, and trypsin was neutralized using HBSS+. Cells were pelleted by centrifugation at $300 \mathrm{~g}$ and washed 3 times with HBSS+. Cells were fixed in 4\% PFA for 20-30 minutes at $37^{\circ} \mathrm{C}$. After fixation, HBSS+ was added to the tube prior to centrifugation to minimize cell loss. Cells were centrifuged at $300 \mathrm{~g}$ and washed 3 times with HBSS. Antibodies were diluted in HBSS+ and cells were stained with either appropriate IgG controls or a combination of rat anti-C3aR, 1:500 (R\&D Systems, MAB10417), mouse antiGlut1 1:1000 (Thermo Fisher Scientific, MA1-37783), and goat antiVE-cadherin 1:1000 (R\&D Systems, MAB9381). Cells were incubated in antibody solution on a benchtop rotator for 30 minutes, then washed 3 times with HBSS+ and incubated in appropriate secondary antibodies for 30 minutes at room temperature in a benchtop rotator. Cells were washed in HBSS +3 times prior to flow cytometry analysis.

Quantitative RT-PCR. RNA was extracted from cells using the RNeasy Micro kit (QIAGEN, 74004). Reverse transcription was performed using the iScript Reverse Transcription Supermix (Bio-Rad, 1708840) according to the manufacturer's protocol. All RNA isolated from cell pellets was converted into cDNA. Quantitative RT-PCR was performed using iTaq Universal SYBR Green Supermix (Bio-Rad, 1725120) on a CFX384 Touch Real-Time PCR Detection System.
Immunostaining and image analysis. Cultured cells were fixed with $4 \%$ PFA for 20 minutes at $37^{\circ} \mathrm{C}$. Samples were washed with PBS and then blocked and permeabilized with PBS containing 0.4\% Triton $\mathrm{X}-100,4 \%$ donkey serum, and 1\% BSA for 30 minutes. Samples were incubated in blocking solution containing primary antibody overnight at $4^{\circ} \mathrm{C}$. Depending on the experiment, primary antibodies were used as follows: rabbit anti-pMLC2 S19 (Cell Signaling Technology, 3671), goat anti-hVE-cadherin (R\&D Systems, AF938), or Phalloidin CruzFluor 555 (Santa Cruz Biotechnology, sc-363794). All images were taken on a Leica TCS laser confocal microscope at $40 \times$ or $63 \times$ under oil immersion, with a $Z$-step of $0.5 \mu \mathrm{m}$ over a total range of $5 \mu \mathrm{m}$. MFI was normalized to cell number per image, and each condition consisted of 8-10 images ( $n=250-300$ cells).

For mouse brain analysis, mice were perfused with $4 \%$ PFA, followed by postfixation in $4 \%$ PFA overnight at $4^{\circ} \mathrm{C}$, and then transferred into $30 \%$ sucrose solution until sectioning. Sagittal brain sections $(30 \mathrm{~mm})$ were cut on a sliding microtome and stored at $-20^{\circ} \mathrm{C}$ in cryoprotectant. After washing in PBS, sections were blocked with PBS containing $0.4 \%$ Triton $\mathrm{X}-100,4 \%$ donkey serum, and 1\% BSA for 30 minutes, and then incubated in blocking solution containing primary antibody overnight at $4^{\circ} \mathrm{C}$. Depending on the experiment, primary antibodies were used as follows: rabbit anti-GFAP (MilliporeSigma, G9269), rat anti-C3 (Hycult, 10129042), rat anti-C3aR (Hycult, 10130173), anti-CD106 (BioLegend, 305802), rat anti-CD31 (BD Biosciences, 550274), goat anti-mVE-cadherin (AF1002), goat anti-PDGFR- $\beta$ (R\&D Systems, AF 1042), mouse anti-Glut1 (Thermo Fisher Scientific, MA1-37783), and rabbit anti-Col IV (Abcam, ab6586). After primary antibody staining, sections were washed in $1 \times$ PBS 3 times and stained with appropriate secondary antibodies for 1-2 hours and washed again before mounting.

For quantification of VCAM1 in the mouse cortex and hippocampus, sections were stained with CD31 and VCAM1, then fluorescent signal was scanned using an EVOS FL Auto system at 10×. Images were then processed by Image $(\mathrm{NIH})$ and background was subtracted before quantification. Total VCAM1-positive signal was quantified as percentage area for each region, hippocampus or cortex. Colocalization of this signal with CD31 was confirmed for accuracy.

For quantification of percentage occupancy of C3 in astrocytes, $Z$-stacks ( $\sim 30 \mu \mathrm{m}$ thick with $0.5 \mu \mathrm{m}$ step size) were taken under $40 \mathrm{x}$ oil immersion, with labeling for $\mathrm{C} 3$, and analyzed using the "Spots" feature of IMARIS 9.2.1 software. Spots were generated automatically for C3 representation. Subsequently, $Z$-stacks were analyzed using the "Co-loc" feature where the $\mathrm{GFAP}^{+}$signal was used to mask astrocyte outlines and thresholds applied to remove background. Data were then recorded as percentage of region of interest (ROI) $\left(\mathrm{GFAP}^{+}\right.$signal mask) occupied by the C3 (Spots) signal. Eight images spanning CA1CA3 were taken across 5 mice per group.

For quantification of average vessel cross-sectional area $Z$-stacks $(\sim 30 \mu \mathrm{m}$ thick with $0.5 \mu \mathrm{m}$ step size) were taken under $40 \times$ oil immersion, with labeling for Col IV. Images were first analyzed using the "Surfaces" feature of IMARIS 9.2.1 software to generate 3D reconstruction of the vessel and a total vessel volume in $\mu \mathrm{m}^{3}$. Subsequently, using the "Filaments" function, the total vessel length per image was estimated by calculating individual vessel branch measurements in $\mu \mathrm{m}$. Average cross-sectional area was determined by the proportional measurement of total vessel volume by total vessel length per image. Six images spanning CA1-CA3 were taken across 5 mice per group. 
For quantification of tortuous vessel morphology, Z-stacks ( 30 $\mu \mathrm{m}$ thick with $0.5 \mu \mathrm{m}$ step size) were taken under $40 \times$ oil immersion with labeling for CD31. Images were manually quantified by counting the number of corkscrew vessels present in projected $Z$-stack images. Images were projected in Leica LAS X software and manually scrolled through to count the number of corkscrew features in hippocampal vasculature. To represent this morphology, representative images were taken under the same imaging parameters but under $63 \times$ oil immersion. The "Surfaces" function of IMARIS 9.2.1 software was used to generate 3D reconstruction of the CD31 vessel, and the animation tab was used to create movies. Six images spanning CA1-CA3 were taken across 5 mice per group for imaging quantification.

For quantification of microglial reactivity, CD68 colocalization in Iba1 signal was quantified using $Z$-stacks imaged on a Leica confocal microscope with $40 \times$ oil objective, a 1.0 digital zoom, a total thickness of $25 \mu \mathrm{m}$, and $1 \mu \mathrm{m}$ step size. Percentage colocalization of CD68 signal within the masked Iba1 ROI was calculated using the "Co-loc" feature in IMARIS and represented as a fold change of this percentage.

To quantify the volume of hippocampus and entorhinal cortex, mouse frozen brain tissues were serially cut at $50 \mu \mathrm{m}$. Sections containing hippocampus or entorhinal cortex (every sixth section, 300 $\mu \mathrm{m}$ apart) between bregma $+2.1 \mathrm{~mm}$ and bregma $-3.9 \mathrm{~mm}$ to the dorsal end of the hippocampus or entorhinal cortex were stained with $0.25 \%$ cresyl violet solution, dehydrated in ethanol, and then mounted. Slides were imaged using Nanozoomer 2.0-HT system (Hamamatsu), and areas of interest were traced using NDP Viewer software. The volume of the region of interest was quantified using the following formula: volume $=($ sum of area $) \times 0.5 \mathrm{~mm}$.

RNA-Seq analysis. RNA-Seq data containing fold changes and adjusted $P$ values from our previous study (22) were used (GEO: GSE114910). For pathway analyses, differentially expressed genes with $P$ (adjusted) values of less than 0.05 were uploaded into the InnateDB website, and overrepresentation analysis was used to calculate significant terms from the KEGG and REACTOME databases. Selected significant terms were plotted by $P$ value on the basis of their involvement in vascular biology and immune cell infiltration, and representative genes contributing to the hits were listed. For individual gene plots, FPKM values were used and significance was calculated using 1-way ANOVA.
Statistics. All statistical analysis was performed using GraphPad Prism software, version 8.0.2 (GraphPad Software). All data are presented as the mean \pm SEM. Unless otherwise noted, all grouped comparisons were made by 1-way ANOVA with Tukey's correction, and all pairwise comparisons by 2 -sided Student's $t$ tests, depending on experimental design. For all tests, $P$ values of less than 0.05 were considered significant, and those over 0.05 were considered nonsignificant.

Study approval. All animal procedures were performed in accordance with NIH guidelines and with the approval of the Baylor College of Medicine IACUC.

\section{Author contributions}

NEP and HZ conceived of the project and designed the experiments. NEP performed all experiments and data analysis unless otherwise noted. ER provided reagents and technical assistance for IMARIS imaging analysis, performed pathway analysis, and edited the manuscript. AL provided samples and technical assistance and performed brain volumetric tissue analysis. JK provided the C3ar1floxed mice. NEP wrote the manuscript with input and revision from HZ. All authors read and approved the final manuscript.

\section{Acknowledgments}

We are indebted to D. Holtzman (Washington University) for the generous support with the brain volumetric analysis. We are most appreciative to the National Institute on Aging for offering aged C57BL6/J mice that made this work possible. We thank C. Beeton, J. Sederstrom, and the Baylor College of Medicine Cytometry and Cell Sorting Core supported by grant NCI-CA125123 for FACS analysis. We are grateful to N. Aithmitti and B. Contreras for expert technical support and members of the Zheng laboratory for stimulating discussions. This study was supported by grants from the NIH (R01 NS093652, R01 AG020670, R01 AG057509, RF1 AG054111, and RF1 AG062257 to HZ).

Address correspondence to: Hui Zheng, Huffington Center on Aging, Baylor College of Medicine, One Baylor Plaza, Houston, Texas 77030, USA. Phone: 713.798.1568; Email: huiz@bcm.edu. AL's present address: Department of Neurology, Washington University School of Medicine in St. Louis, St. Louis, Missouri, USA.
1. Lindenberger U. Human cognitive aging: corriger la fortune? Science. 2014;346(6209):572-578.

2. Maniega SM, et al. White matter hyperintensities and normal-appearing white matter integrity in the aging brain. Neurobiol Aging. 2015;36(2):909-918.

3. Kalamakis G, et al. Quiescence modulates stem cell maintenance and regenerative capacity in the aging brain. Cell. 2019;176(6):1407-1419.e14 .

4. Miranda CJ, et al. Aging brain microenvironment decreases hippocampal neurogenesis through Wnt-mediated survivin signaling. Aging Cell. 2012;11(3):542-552.

5. Sweeney MD, Kisler K, Montagne A, Toga AW, Zlokovic BV. The role of brain vasculature in neurodegenerative disorders. Nat Neurosci. 2018;21(10):1318-1331.

6. Sun D, McGinn M, Hankins JE, Mays KM, Rolfe A, Colello RJ. Aging- and injury-related differential apoptotic response in the dentate gyrus of the hippocampus in rats following brain trauma. Front Aging Neurosci. 2013;5:95.

7. Dhungana $\mathrm{H}$, et al. Aging aggravates ischemic stroke-induced brain damage in mice with chronic peripheral infection. Aging Cell. 2013;12(5):842-850.

8. Sweeney MD, Sagare AP, Zlokovic BV. Bloodbrain barrier breakdown in Alzheimer disease and other neurodegenerative disorders. Nat Rev Neurol. 2018;14(3):133-150.

9. Marchant NL, et al. The aging brain and cognition: contribution of vascular injury and $\mathrm{\beta} \beta$ to mild cognitive dysfunction. JAMA Neurol. 2013;70(4):488-495.

10. Montagne A, et al. APOE4 leads to blood-brain barrier dysfunction predicting cognitive decline. Nature. 2020;581(7806):71-76.

11. Yousef $\mathrm{H}$, et al. Aged blood impairs hippocampal neural precursor activity and activates microglia via brain endothelial cell VCAM1. Nat Med.
2019;25(6):988-1000.

12. Perner C, et al. Plasma VCAM1 levels correlate with disease severity in Parkinson's disease. J Neuroinflammation. 2019;16(1):94.

13. Dulken BW, et al. Single-cell analysis reveals $\mathrm{T}$ cell infiltration in old neurogenic niches. Nature. 2019;571(7764):205-210.

14. Gate D, et al. Clonally expanded CD8 T cells patrol the cerebrospinal fluid in Alzheimer's disease. Nature. 2020;577(7790):399-404.

15. Chen MB, et al. Brain endothelial cells are exquisite sensors of age-related circulatory cues. Cell Rep. 2020;30(13):4418-4432.e4.

16. Katsimpardi L, et al. Vascular and neurogenic rejuvenation of the aging mouse brain by young systemic factors. Science. 2014;344(6184):630-634.

17. Baker DJ, Petersen RC. Cellular senescence in brain aging and neurodegenerative diseases: evidence and perspectives. JClin Invest. 
2018;128(4):1208-1216.

18. Stephan AH, Barres BA, Stevens B. The complement system: an unexpected role in synaptic pruning during development and disease. Annu Rev Neurosci. 2012;35:369-389.

19. Shi $Q$, et al. Complement C3 deficiency protects against neurodegeneration in aged plaque-rich APP/PS1 mice. Sci Transl Med. 2017;9(392):eaaf6295.

20. Shi Q, et al. Complement C3-deficient mice fail to display age-related hippocampal decline. JNeurosci. 2015;35(38):13029-13042.

21. Lian $\mathrm{H}$, et al. NFkappaB-activated astroglial release of complement $\mathrm{C} 3$ compromises neuronal morphology and function associated with Alzheimer's disease. Neuron. 2015;85(1):101-115.

22. Litvinchuk A, et al. Complement C3aR inactivation attenuates tau pathology and reverses an immune network deregulated in tauopathy models and Alzheimer's disease. Neuron. 2018;100(6):1337-1353.e5.

23. Hong S, et al. Complement and microglia mediate early synapse loss in Alzheimer mouse models. Science. 2016;352(6286):712-716.

24. Boire A, Zou Y, Shieh J, Macalinao DG, Pentsova E, Massagué J. Complement component 3 adapts the cerebrospinal fluid for leptomeningeal metastasis. Cell. 2017;168(6):1101-1113.e13.

25. Schraufstatter IU, Trieu K, Sikora L, Sriramarao P, DiScipio R. Complement c3a and c5a induce different signal transduction cascades in endothelial cells. J Immunol. 2002;169(4):2102-2110.

26. Boisvert MM, Erikson GA, Shokhirev MN, Allen $\mathrm{NJ}$. The aging astrocyte transcriptome from multiple regions of the mouse brain. Cell Rep. 2018;22(1):269-285.

27. Swartzlander DB, et al. Concurrent cell typespecific isolation and profiling of mouse brains in inflammation and Alzheimer's disease. JCI Insight. 2018;3(13):e121109.

28. Boulay AC, Saubaméa B, Declèves X, Cohen-Salmon M. Purification of mouse brain vessels. J Vis Exp. 2015;(105):e53208.

29. Wu F, et al. Complement component C3a plays a critical role in endothelial activation and leukocyte recruitment into the brain. J Neuroinflammation. 2016;13:23.

30. Van Beek J, et al. Expression of receptors for complement anaphylatoxins $\mathrm{C} 3 \mathrm{a}$ and $\mathrm{C} 5 \mathrm{a}$ following permanent focal cerebral ischemia in the mouse. Exp Neurol. 2000;161(1):373-382.

31. Ritzel RM, et al. Age-associated resident memory CD8 $\mathrm{T}$ cells in the central nervous system are primed to potentiate inflammation after ischemic brain injury. J Immunol. 2016;196(8):3318-3330.

32. Pi J, et al. Apln-CreERT:mT/mG reporter mice as a tool for sprouting angiogenesis study. $B M C$ Ophthalmol. 2017;17(1):163.

33. Thore CR, Anstrom JA, Moody DM, Challa VR, Marion MC, Brown WR. Morphometric analysis of arteriolar tortuosity in human cerebral white matter of preterm, young, and aged subjects. J Neuropathol Exp Neurol. 2007;66(5):337-345.

34. Bullitt E, et al. Vessel tortuosity and brain tumor malignancy: a blinded study. Acad Radiol. 2005;12(10):1232-1240.

35. Hu J, et al. Inhibition of soluble epoxide hydrolase prevents diabetic retinopathy. Nature. 2017;552(7684):248-252.

36. Shaftel SS, Carlson TJ, Olschowka JA, Kyrkanides S, Matousek SB, O'Banion MK. Chronic interleukin-1beta expression in mouse brain leads to leukocyte infiltration and neutrophil-independent blood brain barrier permeability without overt neurodegeneration. J Neurosci. 2007;27(35):9301-9309.

37. Wu MC, et al. The receptor for complement component $\mathrm{C} 3$ a mediates protection from intestinal ischemia-reperfusion injuries by inhibiting neutrophil mobilization. Proc Natl Acad Sci U S A 2013;110(23):9439-9444.

38. Shi Y, et al. Rapid endothelial cytoskeletal reorganization enables early blood-brain barrier disruption and long-term ischaemic reperfusion brain injury. Nature Communications. 2016;7:10523.

39. Tietz S, Engelhardt B. Brain barriers: crosstalk between complex tight junctions and adherens junctions. JCell Biol. 2015;209(4):493-506.
40. Quell KM, et al. Monitoring C3aR expression using a floxed tdTomato-C3aR reporter knock-in mouse. JImmunol. 2017;199(2):688-706.

41. Kisanuki YY, Hammer RE, Miyazaki J, Williams SC, Richardson JA, Yanagisawa M. Tie2-Cre transgenic mice: a new model for endothelial cell-lineage analysis in vivo. Dev Biol. 2001;230(2):230-242.

42. Tanaka Y, Adams DH, Hubscher S, Hirano H, Siebenlist U, Shaw S. T-cell adhesion induced by proteoglycan-immobilized cytokine MIP-1 beta. Nature. 1993;361(6407):79-82.

43. Kolev M, et al. Diapedesis-Induced integrin signaling via LFA-1 facilitates tissue immunity by inducing intrinsic complement $\mathrm{C} 3$ expression in immune cells. Immunity. 2020;52(3):513-527.e8

44. Nation DA, et al. Blood-brain barrier breakdown is an early biomarker of human cognitive dysfunction. Nat Med. 2019;25(2):270-276.

45. Montagne A, et al. Blood-brain barrier breakdown in the aging human hippocampus. Neuron 2015;85(2):296-302.

46. Iadecola C. Dangerous leaks: blood-brain barrier woes in the aging hippocampus. Neuron. 2015;85(2):231-233.

47. Banks WA, et al. Lipopolysaccharide-induced blood-brain barrier disruption: roles of cyclooxygenase, oxidative stress, neuroinflammation, and elements of the neurovascular unit. J Neuroinflammation. 2015;12:223.

48. Yang AC, et al. Physiological blood-brain transport is impaired with age by a shift in transcytosis. Nature. 2020;583(7816):425-430.

49. Marsh SE, et al. The adaptive immune system restrains Alzheimer's disease pathogenesis by modulating microglial function. Proc Natl Acad Sci U S A. 2016;113(9):E1316-E1325.

50. Laurent $\mathrm{C}$, et al. Hippocampal $\mathrm{T}$ cell infiltration promotes neuroinflammation and cognitive decline in a mouse model of tauopathy. Brain. 2017;140(1):184-200.

51. Faraco G, et al. Dietary salt promotes cognitive impairment through tau phosphorylation. Nature. 2019;574(7780):686-690. 